\title{
Genome-Wide Association Mapping of Loci for Resistance to Stripe Rust in North American Elite Spring Wheat Germplasm
}

\author{
Jayfred Gaham Godoy, Sheri Rynearson, Xianming Chen, and Michael Pumphrey ${ }^{\dagger}$
}

First, second, and fourth authors: Department of Crop and Soil Sciences, Washington State University, Pullman 99164-6420; and third author: United States Department of Agriculture-Agricultural Research Service and Department of Plant Pathology, Washington State University, Pullman 99164-6430.

Accepted for publication 22 September 2017.

\begin{abstract}
Stripe rust, caused by Puccinia striiformis f. sp. tritici, is a major yieldlimiting foliar disease of wheat (Triticum aestivum) worldwide. In this study, the genetic variability of elite spring wheat germplasm from North America was investigated to characterize the genetic basis of effective all-stage and adult plant resistance (APR) to stripe rust. A genome-wide association study was conducted using 237 elite spring wheat lines genotyped with an Illumina Infinium $90 \mathrm{~K}$ single-nucleotide polymorphism array. All-stage resistance was evaluated at seedling stage in controlled conditions and field evaluations were conducted under natural disease pressure in eight environments across

Washington State. High heritability estimates and correlations between infection type and severity were observed. Ten loci for race-specific all-stage resistance were confirmed from previous mapping studies. Three potentially new loci associated with race-specific all-stage resistance were identified on chromosomes 1D, 2A, and 5A. For APR, 11 highly significant quantitative trait loci (QTL) (false discovery rate $<0.01$ ) were identified, of which 3 QTL on chromosomes $3 \mathrm{~A}, 5 \mathrm{D}$, and 7A are reported for the first time. The QTL identified in this study can be used to enrich the current gene pool and improve the diversity of resistance to stripe rust disease.
\end{abstract}

Stripe rust of wheat, caused by the fungus Puccinia striiformis $\mathrm{f}$. sp. tritici Erikss., is one of the most prevalent diseases limiting global wheat production. As with many airborne pathogens, long-distance dispersal in the air enables spread of $P$. striiformis $\mathrm{f}$. sp. tritici across different geographic regions. The disease is so widespread that it has been reported in more than 60 wheat-producing countries and all continents except Antarctica (Chen 2005). In these regions, stripe rust damage can be as high as $70 \%$ when susceptible of cultivars are planted and climatic conditions favoring early infection and extended duration of disease occurrence. Aside from reduced yields, infected plants produce poor quality seed with low vigor and poor emergence when planted the following season. Although proper timing of application of effective fungicides has prevented major yield losses, this approach is a financial burden for growers, especially in areas with low grain yields. In regions with high disease pressure, substantial losses are still prevalent on highly susceptible varieties despite fungicide application. Breeding of resistant cultivars coupled with their timely deployment is still the most economical and environmentally friendly approach to prevent yield losses caused by stripe rust.

Concerted efforts by many wheat-breeding institutions to create stripe rust-resistant wheat varieties have been successful but have been relatively short lived due to the rapid evolution of new $P$. striiformis $\mathrm{f}$. sp. tritici races (Line and Chen 1995; Line and Qayoum 1992). To date, there are approximately 78 officially named $\mathrm{Yr}$ genes $(\mathrm{Yrl}$ to $\mathrm{Yr} 78)$, and 67 with temporary $Y r$ designations (McIntosh et al. 2013; Xiang et al. 2016). However, only a small number of these genes have been introduced into wheat varieties and breeding lines (Chen et al. 2014).

Resistance to stripe rust is usually characterized as either allstage resistance (also called seedling resistance) or adult-plant resistance (APR). All-stage resistance is race specific and expressed from seedling to adult-plant stage (Chen 2005). This type of

${ }^{\dagger}$ Corresponding author: M. Pumphrey; E-mail: m.pumphrey@wsu.edu

*The $\boldsymbol{e}$-Xtra logo stands for "electronic extra" and indicates that six supplementary figures and two supplementary files are published online.

(c) 2018 The American Phytopathological Society resistance generally displays a strong hypersensitive response and interacts with the pathogen in a gene-for-gene manner. Varieties having only all-stage resistance often become susceptible soon after their release (Line and Qayoum 1992). Examples of major $Y r$ genes that have become ineffective include $Y r 2, \operatorname{Yr} 6, \operatorname{Yr} 7, \operatorname{Yr} 8, \operatorname{Yr} 9, \mathrm{Yr} 17$, and $Y r 27$ (Singh et al. 2008). APR resistance is usually quantitative and considered less affected by specific pathogen races and generally more durable. Plants having only APR are susceptible or moderately susceptible at seedling stages but become more resistant as the plant develops. High-temperature adult-plant (HTAP) resistance is a type of APR that expresses later in the growing season as temperature increases and plants grow older (Milus and Line 1986a,b; Qayoum and Line 1985). Pyramiding both types of resistance into wheat varieties would provide a more durable and high level of resistance against stripe rust throughout the entire growing season (Chen 2005, 2013).

Quantitative trait locus (QTL) mapping in biparental populations has been the most widely used strategy to identify genomic regions associated with stripe rust resistance. Rosewarne et al. (2013) summarized approximately 140 QTL distributed across 49 chromosomal regions conferring resistance to stripe rust that were reported in over 30 publications during the last 10 years. However, because these QTL were identified in biparental populations, they may represent only a small fraction of the natural variation in diverse germplasm. Genomewide association analysis (GWAS) can be used to capture a larger portion of the natural variation present in a collection of different genotypes, while localizing associations to much smaller genomic regions by utilizing more recombination events compared with recombinant inbred lines (RIL) or doubled-haploid (DH) populations (Nordborg and Weigel 2008). This improvement over biparental QTL mapping, plus the time saved by not needing to make crosses and generate populations, may allow for broader and more rapid tracking of QTL in breeding programs. GWAS has been successfully used to identify QTL for grain yield (Edae et al. 2014; Sukumaran et al. 2015), Hessian fly ( $\mathrm{Li}$ et al. 2013), and stripe rust resistance in synthetic wheat (Zegeye et al. 2014), spring wheat (Jighly et al. 2015), and winter wheat (Bulli et al. 2016; Naruoka et al. 2015), among others. 
Races of $P$. striiformis f. sp. tritici in North America are among the most diverse in all wheat-producing regions. The Pacific Northwest (PNW) states of the United States plus California and southeastern British Columbia, Canada provide a favorable environment (cool and wet springs and dry and cool summers) for pathogen survival and development (Sharma-Poudyal et al. 2014). Growing both winter wheat and spring wheat in these regions also provides a continuous supply of inoculum from living plants (Chen 2005). Stripe rust outbreaks in neighboring states and even across the Rocky Mountains toward the Midwest of the United States may originate from these areas (Xia et al. 2016). Spring wheat across North America has experienced more widespread stripe rust damage over the past decade, and it is important to identify new sources of resistance for wheat-breeding programs in this region.

In this study, we used an association panel composed of elite spring wheat genotypes from public breeding programs in North America, which was genotyped with the Illumina Infinium 90K single-nucleotide polymorphism (SNP) assay, to address the following objectives: (i) assess the diversity of disease response in the population, (ii) conduct an association mapping (AM) study using genome-wide SNP markers to identify chromosomal regions associated with stripe rust response in both seedling and adult-plant stages, and (iii) compare the identified marker-trait associations (MTA) with previously identified QTL.

\section{MATERIALS AND METHODS}

Genetic materials. A population consisting of 237 elite spring wheat genotypes was used. The panel was assembled by the Triticeae Coordinated Agriculture Program (TCAP) (Fig. 1). It included cultivars, advanced breeding lines, and important parental lines from six public wheat-breeding programs in the United States, two breeding programs in Canada, and the International Maize and Wheat Improvement Center (CIMMYT). The cultivar Lemhi
(CI011415) was added to the panel as a susceptible check. Lemhi carries the all-stage resistance gene $Y r 21$ that is ineffective against almost all $P$. striiformis f. sp. tritici races in the United States. The TCAP elite spring wheat AM panel was also used in previous GWAS for resistance to stem rust (Bajgain et al. 2015) and sawfly (Varella et al. 2015).

Stripe rust screening. Seedlings of the 237 genotypes were tested for their reaction to $P$. striiformis $\mathrm{f}$. sp. tritici isolates PSTv-4 and PSTV-14 (Wan and Chen 2014) under controlled greenhouse conditions. PSTv-4 (vir/avir formula: $Y r 1, Y r 6, Y r 9, Y r 17$, Yr27, YrSP, Yr76/Yr5, Yr7, Yr8, Yr10, Yr15, Yr24, Yr32, Yr43, Yr44, $Y r T r 1$, and $Y r E x p 2)$ and PSTv-14 (vir/avir formula: $Y r 1, Y r 6, Y r 7$, Yr8, Yr9, Yr17, Yr27, Yr43, Yr44, YrTr1, YrExp2, Yr76/Yr5, Yr10, Yr15, Yr24, Yr32, and YrSP) are predominantly found in the PNW and California. Three seeds of each genotype were planted per well in a 96-well tray filled with number 1 Sunshine mix growing medium (Sun Gro Horticulture, Bellevue, WA). Each experiment included the susceptible check variety Avocet $\mathrm{S}$ and the set of $\mathrm{Yr}$ single-gene differential stocks in the Avocet $S$ background for differentiating P. striiformis f. sp. tritici races (Wan and Chen 2014). The trays were regularly watered and kept in a rust-free greenhouse at $20^{\circ} \mathrm{C}$ with $50 \%$ relative humidity (RH). Ten-day-old seedlings at approximately the two-leaf stage were inoculated with urediniospores of each race mixed with talcum powder. Inoculated plants were incubated in a dark dew chamber for $24 \mathrm{~h}$ at $10^{\circ} \mathrm{C}$ and $100 \%$ $\mathrm{RH}$, and then moved to a regular greenhouse with a diurnal cycle of 8 and $16^{\circ} \mathrm{C}$ (day and night temperatures, respectively) and a $16-\mathrm{h}$ photoperiod. Reactions to $P$. striiformis f. sp. tritici infection were scored 18 to 20 days after inoculation using a 0 -to- 9 scale $(0$ to $3=$ resistant, 4 to $6=$ intermediate, and 7 to $9=$ susceptible) for infection type (IT) (Line and Qayoum 1992; McNeal et al. 1971).

The panel was evaluated for stripe rust reaction under natural disease conditions in adult plant stages at three different locations: Spillman (PLM) and Whitlow (WLW) farms in Pullman, WA and at

TCAP Elite Spring AM Panel

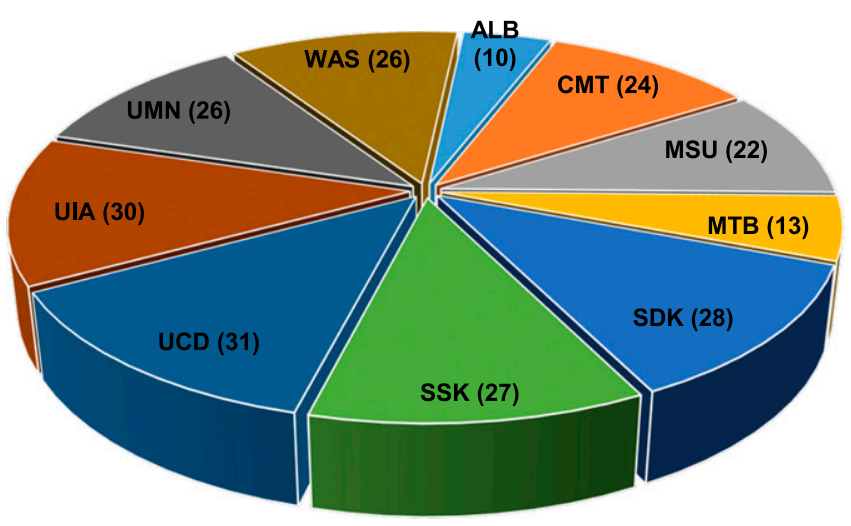

Breeding program:

\begin{tabular}{|c|c|}
\hline ALB - University of Saskatchewan (Alberta) & SSK - University of Saskatchewan (Saskatoon) \\
\hline CMT - CIMMYT & UCD - University of California (Davis) \\
\hline MSU - Montana State University & UIA - University of Idaho (Aberdeen) \\
\hline MTB - University of Saskatchewan (Manitoba) & UMN - University of Minnesota \\
\hline SDK - South Dakota State University & WAS - Washington State University \\
\hline
\end{tabular}

Fig. 1. Distribution of 237 elite spring wheat genotypes in the Triticeae Coordinated Agriculture project (TCAP) association mapping (AM) panel by their respective breeding programs. 
Mount Vernon, WA (MTV). The field trials were conducted from 2012 to 2014 for all locations except for Spillman, which was omitted in 2013. Thus, in total, eight year-location combinations were conducted, hereafter referred to as "environments". All fields were maintained using prescribed management practices for fertilizer application and weed control for spring wheat to provide optimum growing conditions. Supplemental irrigation was used in the Spillman trials, whereas both Whitlow and Mount Vernon trials were rain fed. In each environment, $5 \mathrm{~g}$ of seed from each genotype was planted in nonreplicated single-row plots $1 \mathrm{~m}$ in length and spaced $0.35 \mathrm{~m}$ apart. Lemhi was planted every 20 rows and in spreader rows planted surrounding the plots to ensure continuous production of inoculum sufficient for uniform disease development. Data collection commenced as soon as disease severity (SEV) $(>90 \%)$ became relatively uniform on Lemhi at each environment, approximately between heading (Zadok's 50) and grain filling (Zadok's 80) stages. IT was scored based on a 0-to-9 scale similar to greenhouse evaluation and SEV as visual estimate of the percentage of leaf area infected (0 to 100) in the row (Line and Qayoum 1992). Disease was scored at least twice and only data from the rating with the highest average disease pressure across all genotypes per environment were used in GWAS.

Statistical analyses. Mean, standard deviation, and minimum and maximum values of disease scores within and across environments were calculated using JMP Genomics 6.0 (SAS Institute Inc. 2007). Pearson correlation coefficients for both IT and SEV values across environments were calculated using the PROC CORR procedure in SAS v9.3. This correlation was done to evaluate the pattern of disease response among the genotypes at different environments. Broad-sense heritability $\left(H^{2}\right)$ was estimated for both IT and SEV values across environments using the variance components obtained from the random effects model computed using PROC MIXED procedure in SAS v9.3. Best linear unbiased estimate (BLUE) values across the eight environments were also calculated for IT and SEV, considering genotypes as fixed effects. BLUE values were used to compare different GWAS models. The proportions of variation $\left(R^{2}\right)$ in IT and SEV explained by population structure were assessed using multiple regression of BLUE across environments on the STRUCTURE membership coefficient matrix (Q).

DNA extraction, SNP marker genotyping, and molecular analyses. Fresh leaves from each genotype were harvested at the three-leaf stage and genomic DNA was extracted using a BioSprint96 machine following the BioSprint 96 plant DNA extraction protocol (https://www.qiagen.com/us/). DNA samples were genotyped at the United States Department of Agriculture-Agricultural Research Service genotyping laboratory in Fargo, ND. SNP genotyping was implemented using the Illumina iSelect $90 \mathrm{~K}$ SNP assay, as described by Wang et al. (2014). Monomorphic and low-quality SNP were first discarded using Genome Studio software v2011.1. The default clustering algorithm was used to select biallelic SNP that showed distinct clusters that correspond to $\mathrm{AA}, \mathrm{AB}$, and $\mathrm{BB}$ genotypes. Manual curation was further conducted on SNP allele clusters that were not clearly determined by the clustering algorithm (Cavanagh et al. 2013). Markers with a minor allele frequency $(\mathrm{MAF})<0.05$ were excluded to avoid the high statistical power required to make meaningful statements about associations with rare alleles (Tabangin et al. 2009). In total, 19,192 high-quality SNP markers were selected and ordered based on the rescaled map positions of hexaploid wheat $90 \mathrm{~K}$ SNP consensus map (Wang et al. 2014). The B genome had the highest number of polymorphic markers $(9,803)$, followed by the A genome with 7,593 SNP. The D genome was the least covered, with 1,796 polymorphic SNP markers. These polymorphic SNP were used in the subsequent association analyses. Additional genotypic data were generated for molecular markers linked to loci conferring resistance to multiple pathogens. KaspYr5-6121 and KaspYr5-4096 assays are flanking markers for the stripe rust resistance gene $\operatorname{Yr} 5$ (Naruoka et al. 2016). Simple sequence repeat (SSR) marker barc8 (http://maswheat.ucdavis.edu/protocols/Yr15/index.htm) is positioned 9 centimorgans (cM) distal to $\operatorname{Yr} 15$. KaspLr34 assay
(http://maswheat.ucdavis.edu/protocols/Yr15/index.htm, wMAS000003) was designed around a 3-bp indel in exon 11 of the Lr34/Yrl8 gene (Lagudah et al. 2009). KaspSr2 assay was developed based on Mago et al. (2011). A marker for stem rust resistance gene Sr28 (Bajgain et al. 2015) was included because our previous studies indicated that stripe rust resistance genes also mapped in this region.

Linkage disequilibrium and population structure. Linkage disequilibrium (LD) among mapped markers was analyzed using JMP Genomics 6.0 (SAS Institute Inc. 2007) by computing the squared allele-frequency correlations $\left(r^{2}\right)$, according to Weir (1996). Only those pairwise marker combinations with significant $r^{2}(P<0.01)$ were included in the analysis. The unlinked $r^{2}$ and syntenic $r^{2}$ were estimated separately for unlinked loci (distance $>50 \mathrm{cM}$ ) on the same chromosome. A critical $r^{2}$ value beyond which LD is due to physical linkage was determined by taking the 95th percentile of unlinked markers (Breseghello and Sorrells 2006). Locally weighed polynomial regression (LOESS)-based curves were then fitted on scatter plots of $r^{2}$ values against distance among markers. The intersection of the LOESS curve fit and critical value of $r^{2}$ was considered as the estimate of the extent of LD in the chromosome.

A subset of 749 SNP markers spaced 3 to $4 \mathrm{cM}$ apart was sampled from all chromosomes for population structure analysis using the Bayesian model-based clustering program STRUCTURE V2.3 (Pritchard et al. 2000) to estimate the number of subpopulations $(K)$. Simulations were run for 10,000 burn-in iterations followed by 100,000 Monte Carlo Markov Chain replicates. The number of $K$ was set from 1 to 10 , with 10 independent runs made for each $K$. The results from structure were visualized using STRUCTURE HARVESTER (Earl and von Holdt 2012) and the $K$ number of subpopulations was determined using the ad hoc criterion described by Evanno et al. (2005). The relative kinship matrix was constructed using JMP Genomics 6.0 (SAS Institute Inc. 2007), and negative estimates between any pair of lines were set to 0 .

Association analysis. In total, 19,192 SNP markers were used in the association analyses. MTA were tested using a mixed linear model (MLM) approach that included BLUE, marker data, kinship (K matrix), and probability of membership of each line (Q matrix) for each trait (Yu et al. 2006). The K and Q (from STRUCTURE) matrices were assigned as random and fixed components, respectively, in MLM. Association tests were run using the software Genomic Association and Prediction Integrated Tool (GAPIT) implemented in $\mathrm{R}$ software (Lipka et al. 2012). Model comparison was conducted to select the best model for detecting MTA on each trait following previously recommended procedures for unified mixed-model AM (Edae et al. 2014; Sukumaran et al. 2015; Yu et al. 2006; Zhu et al. 2008). BLUE for each trait were used in model comparisons considering their Q-Q plots. The different models tested were: K (kinship) only, Q+K (population structure and kinship), and $\mathrm{P}+\mathrm{K}$ (principal component and kinship). The best models were selected based on their respective Q-Q plots. MTA $P$ values for all traits in the combined data set were used for model comparison. Significant MTA were described as $P$ values and the magnitude of QTL effects, $R^{2}$, were evaluated by comparing to a "null allele" (missing positive allele) model for each locus (Breseghello and Sorrells 2006).

To assess whether the MTA detected in the current study had been identified previously or not, we projected the MTA to an integrated map developed by Maccaferri et al. (2015). This map included chromosomal positions of previously identified $Y r$ genes and QTL and their confidence intervals. The integrated map was compiled from different wheat consensus maps developed since 2005 using different marker platforms. These included the 9K (Cavanagh et al. 2013) and 90K SNP (Wang et al. 2014) consensus maps, tetraploid consensus map (Maccaferri et al. 2015), Synthetic $\times$ Opata DH GBS map (Saitenac et al. 2013), Diversity Array Technology (DArT) integrated map (http://www.diversityarrays.com/search/node/Wheat\%20DArT \%20map), 2004 SSR consensus map (Somers et al. 2004), and Synthetic $\times$ Opata ITMI BARC SSR map (Song et al. 2005). The chromosomal locations of published $\mathrm{Yr}$ resistance genes and QTL 
were expressed as relative percent length distances to the total chromosome length of the closet flanking markers, which were used as confidence intervals (Maccaferri et al. 2015).

\section{RESULTS}

Phenotypic variation for response to $\boldsymbol{P}$. striiformis f. sp. tritici infection. Under controlled greenhouse conditions, IT scores for both races followed a bimodal distribution (one mode at IT 1 and 2 and another mode at IT 7 and 8) and were skewed toward susceptibility (Fig. 2). For PSTv-4, only $22 \%$ of the genotypes were resistant whereas $68 \%$ were susceptible, and the remaining $10 \%$ showed intermediate responses (IT 3 to 6). A similar distribution was observed for PSTv-14, in which 18, 70, and $12 \%$ of the genotypes had resistant, susceptible, and intermediate reactions, respectively. Under natural disease pressure over eight environments, IT scores were, in general, normally distributed (Supplementary Fig. S1). At these field locations, severe stripe rust was observed and the susceptible check Lemhi always had IT scores of 8 to 9 and SEV of 80 to $100 \%$. The mean IT scores across years in each location and across environments were similar, at approximately IT 5 (Table 1). The mean SEV was higher at Mount Vernon compared with the other environments (Table 1).

Estimates of variance components showed highly significant $(P<$ $0.0001)$ differences for IT and SEV among the genotypes across all environments. Significant differences for environment and genotypeenvironment interactions were also observed but not across all environments, indicating the influence of climatic conditions and different $P$. striiformis f. sp. tritici races on disease development. High $H^{2}$ estimates were observed across years for each environment, ranging from 0.84 to 0.92 for IT and 0.80 to 0.93 for SEV, and even greater for BLUE values across all locations $(0.95$ for both IT and SEV). Pearson correlation coefficients $(r)$ for stripe rust response within and across locations were highly significant $(P<0.0001)$, ranging from 0.60 to 0.89 for IT and 0.54 to 0.84 for SEV (Table 2). Correlations within years at each location were usually higher than across years but lower correlations were observed when comparing IT and SEV values across different locations.

LD decay and population structure. The extent of LD, LD decay rate, and population structure on this TCAP panel were described by Bagjain et al. (2015). In this population, 50\% LD decay was observed within approximately $3 \mathrm{cM}$ genetic distance (Supplementary Fig. S2). Hence, the confidence interval used to establish a QTL in this study was approximately $3 \mathrm{cM}$ on either side of a significant MTA. Markers within this confidence interval were considered part of a single resistance-associated locus. Population structure and kinship analyses revealed that the 237 genotypes can be subdivided into two subpopulations (Q1 and Q2), which generally corresponded to the geographic location of the different breeding programs from which the genotypes were developed (Supplementary Fig. S3). Subpopulation Q1 was composed of 127 genotypes of breeding programs from the western United States and CIMMYT, whereas 110 genotypes from breeding programs in the Northern Plains of the United States plus Canada made up subpopulation Q2. Multiple regression analysis further showed association of population structure on IT $\left(R^{2}=0.126\right)$ and $\operatorname{SEV}\left(R^{2}=0.127\right)$. A similar trend was also observed in the boxplots of BLUE values for IT and SEV (Supplementary Fig. S4). Genotypes in subpopulation Q1 had significantly (Tukey's $P<0.0001$ ) lower IT and SEV compared with genotypes in subpopulation Q2. The significant influence of population structure on stripe rust infection was taken into account in the subsequent GWAS analyses as covariates in the MLM.

MTA for race-specific stripe rust response. In total, 13 MTA across 10 chromosomal regions were significantly $(P<0.001)$ associated with IT score (Table 3 ). The phenotypic variation $\left(R^{2}\right)$ explained by these QTL ranged from 4 to $19 \%$. The largest effect locus for stripe rust resistance at the seedling stage was detected at the 71.64-cM short arm of chromosome 5B $\left(R^{2}=0.19\right)$. This region was tagged by SNP IWA7915 and was associated with IT response to isolate PSTV-14. Three loci were detected at regions of chromosome $1 \mathrm{~A}$ at $29.11 \mathrm{cM}$ (IWB12795), $48.95 \mathrm{cM}$ (IWB20633), and $83.70 \mathrm{cM}$ (IWB56353). These MTA were associated with IT response to PSTv-4 with $R^{2}=0.04$. SNP markers IWB34847 on chromosome 1B at $31.09 \mathrm{cM}$ and IWB35520 on chromosome 1D at $89.58 \mathrm{cM}$ were associated with IT response to PSTV-14, explaining 4 and $5 \%$ of the phenotypic variation, respectively. Two loci, each with $R^{2}=0.05$, were detected on chromosome 2A. SNP markers IWB1593.1 $(123.65 \mathrm{cM})$ and IWB10956 $(139.85 \mathrm{cM})$ were associated with IT scores for PSTV-4 and PSTv-14, respectively. One locus on chromosome 2B at $109.24 \mathrm{cM}$, tagged by SNP marker IWB32165, was associated with resistance to both PSTv-4 $\left(R^{2}=0.04\right)$ and PSTv-14 $\left(R^{2}=0.05\right)$. SNP markers IWB26833 on chromosome 3A at 59.01 $\mathrm{cM}$ and IWA3530 on chromosome 5A at $39.62 \mathrm{cM}$ were both found associated with IT for PSTv-4, with $R^{2}$ values of 0.04 and 0.05 , respectively. Markers IWB25986 (57.07 cM) and IWB74027 (66.08 $\mathrm{cM}$ ) on the short arm of chromosome 6B were associated with PSTv$4\left(R^{2}=0.04\right)$ and PSTv-14 $\left(R^{2}=0.05\right)$, respectively. In addition, significant MTA between the SSR marker linked to $Y r 15$ and IT response to PSTV-4 and PSTv-14 were also detected in the analysis, with $R^{2}=0.06$ (Table 3 ). The KaspSr28 marker was also associated with IT response to PSTv-14 $\left(R^{2}=0.04\right)$. The KaspSr2 markers were not significantly associated with IT and SEV at seedling and adult stages. The MAF for $Y r 5$ was below the allele frequency cutoff value (MAF < 0.05) (Supplementary File S1); hence, these were excluded in the association analyses.

MTA for stripe rust response under field conditions. Marker-trait association analyses were performed for IT and SEV on each of the eight environments and using BLUE. In total, 25 SNP

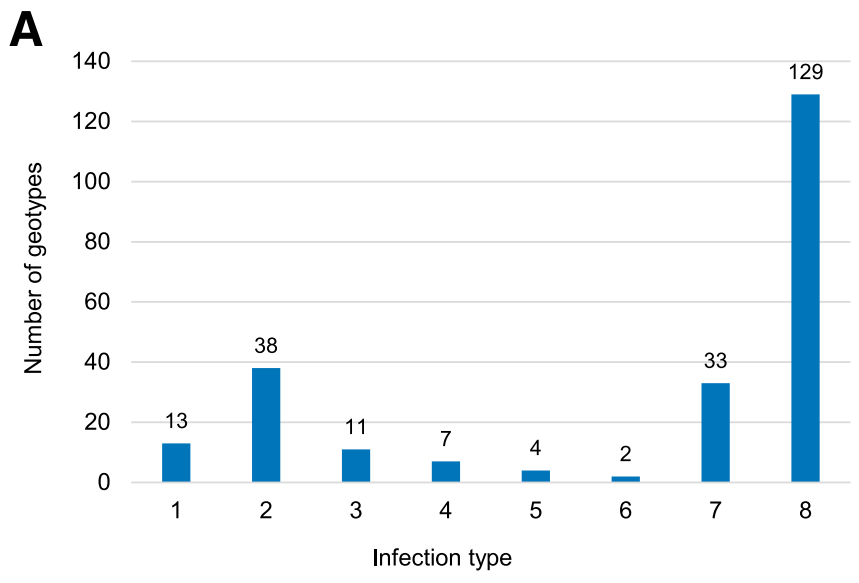

B

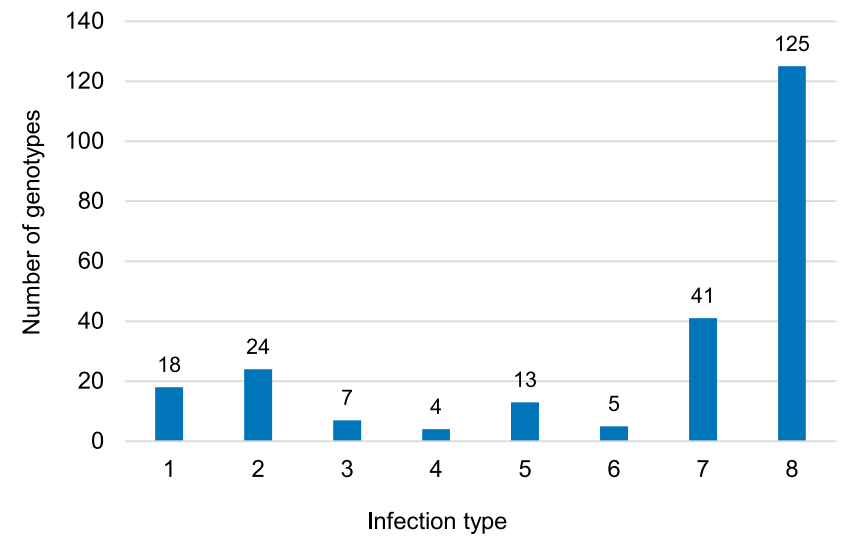

Fig. 2. Distributions of infection type evaluated in the seedling stage for A, PSTv-4 and B, PSTv-14 in the Triticeae Coordinated Agriculture Project (TCAP) association mapping (AM) spring wheat panel. 
markers mapped on 13 chromosomes were significantly associated $(P<0.01)$ with either IT or SEV in at least four environments (Supplementary File S2). Four loci were mapped on chromosome 2A; three on 6B; two each on 2B, 3A, 6A, 7A, and 7B; and one QTL each on chromosomes 2D, 3B, 4B, 5D, and 6D. The magnitude of effects of each locus, expressed as percentage of explained variation $\left(R^{2}\right)$, varied across different environments, ranging between 2 and 12\%. Among these 25 loci, 11 passed the false discovery rate $($ FDR $<0.01)$ threshold in at least one environment (Table 4). QTL nomenclature, chromosome location, probabilities of association with IT and SEV from multienvironment tests, and BLUE values are included. The magnitude of their effects, represented by the maximum $R^{2}$ value, and the frequency of their favorable alleles in the entire panel and across the two subpopulations are also presented.

Of the 11 loci, 6 (located on chromosomes 2A, 2B, 2D, and 3A) were significantly associated with IT and SEV in all environments, including their respective BLUE for IT and SEV. Among these, QYr.tsw-2A.3, QYr.tsw-2A.4, and QYr.tsw-2A.5 were mapped within the 9.41- to 48.44-cM region on the short arm of chromosome $2 \mathrm{~A}$ and associated with SNP markers IWB11136, IWB24342, and IWB65219, respectively. The $R^{2}$ of these three loci for IT ranged from 7 to $8 \%$ and for SEV were $12 \%$ for each. QYr.tsw-2B.2, identified by SNP marker IWB34480, was mapped on the short arm of chromosome $2 \mathrm{~B}$ at $19.95 \mathrm{cM}$ and associated with IT $\left(R^{2}=0.08\right)$ and SEV $\left(R^{2}=0.12\right)$. The locus QYr.tsw-2D (IWB11792) was mapped on chromosome $2 \mathrm{D}$ at $6.75 \mathrm{cM}$ and explained as much as 8 and $12 \%$ of the phenotypic variation in IT and SEV, respectively. SNP marker IWB36635 was associated with QYr.tsw-3A.2 on chromosome $3 \mathrm{~A}$ at $123.01 \mathrm{cM}$, with $R^{2}=0.09$ for IT and $R^{2}=0.12$ for SEV. The frequencies of the favorable alleles for these six loci in the panel were very low $(<10 \%)$ and mostly detected in subpopulation Q1 with genotypes developed in the western United States and CIMMYT. None of these six loci were associated with seedling response to infection with PSTv-4 or PSTv-14.

Other loci were identified on chromosomes 5D, 6B, 6D, and 7A. QYr.tsw-5D was identified by SNP marker IWA2821 on chromosome $5 \mathrm{D}$ at $190.80 \mathrm{cM}$. This locus was significantly associated with IT evaluated from MTV-13, MTV-14, and WLW-13 and BLUE-IT $\left(R^{2}=0.04\right)$. This locus was also associated with SEV at MTV-13, MTV-14, PLM-12, PLM-14, WLW-13, and WLW-14 and BLUE$\operatorname{SEV}\left(R^{2}=0.05\right)$. Two loci were identified on the short arm of chromosome 6B, at $57.07 \mathrm{cM}($ QYr.tsw-6B.4) and $66.08 \mathrm{cM}$ (QYr.tsw-6B.2). QYr.tsw-6B.4 was identified with SNP marker IWB45887, except for IT at MTV-14 and SEV at WLW-12, and this

TABLE 1. Means and ranges for response to Puccinia striiformis f. sp. tritici of 237 elite spring wheat genotypes in eight environments ${ }^{\mathrm{a}}$

\begin{tabular}{|c|c|c|c|c|c|c|c|c|}
\hline \multirow[b]{2}{*}{ Item } & \multicolumn{2}{|c|}{ Mount Vernon } & \multicolumn{2}{|c|}{ Pullman } & \multicolumn{2}{|c|}{ Whitlow } & \multicolumn{2}{|c|}{ Across environments } \\
\hline & IT & SEV & IT & SEV & IT & SEV & IT & SEV \\
\hline Mean & 5.3 & 53.4 & 5.2 & 42.7 & 4.9 & 42.6 & 5.1 & 46.2 \\
\hline Minimum & 0 & 0 & 0 & 0 & 0 & 0 & 0 & 0 \\
\hline Maximum & 9 & 100 & 9 & 90 & 9 & 100 & 9 & 96.7 \\
\hline$\sigma_{G}^{2}$ & $4.82 * * *$ & $635.01 * * *$ & $3.74 * * *$ & $479.46^{* * * *}$ & $2.34 * * *$ & $295.46^{* * * *}$ & $3.41 * * *$ & $438.50 * * *$ \\
\hline$\sigma_{E}^{2}$ & $1.24 * * *$ & 1.00 & $0.99 * * *$ & 1.00 & 1.27 & 1.00 & 1.10 & 1.00 \\
\hline$\sigma_{G x E}^{2}$ & 0.00 & $153.21 * * *$ & 0.00 & $120.55 * * *$ & $0.05^{\mathrm{ns}}$ & $222.90 * * *$ & $0.31 * * *$ & $200.47 * * *$ \\
\hline$\sigma_{e}^{2}$ & $0.24^{\mathrm{ns}}$ & $5.55^{\mathrm{ns}}$ & 0.00 & $13.55^{\mathrm{ns}}$ & $0.19^{\text {ns }}$ & $36.74^{\mathrm{ns}}$ & $0.15^{*}$ & $44.70^{*}$ \\
\hline$H^{2}$ & 0.92 & 0.93 & 0.88 & 0.89 & 0.84 & 0.80 & 0.95 & 0.95 \\
\hline
\end{tabular}

a IT $=$ infection type and SEV $=$ disease severity. Asterisks $*$ and $* * *$ indicate $P<0.05$ and $P<0.001$, respectively, and ns $=$ not significant.

TABLE 2. Pearson's correlation coefficients for infection type (IT) and severity (SEV) response to Puccinia striiformis f. sp. tritici of 237 elite spring wheat genotypes $^{\mathrm{a}}$

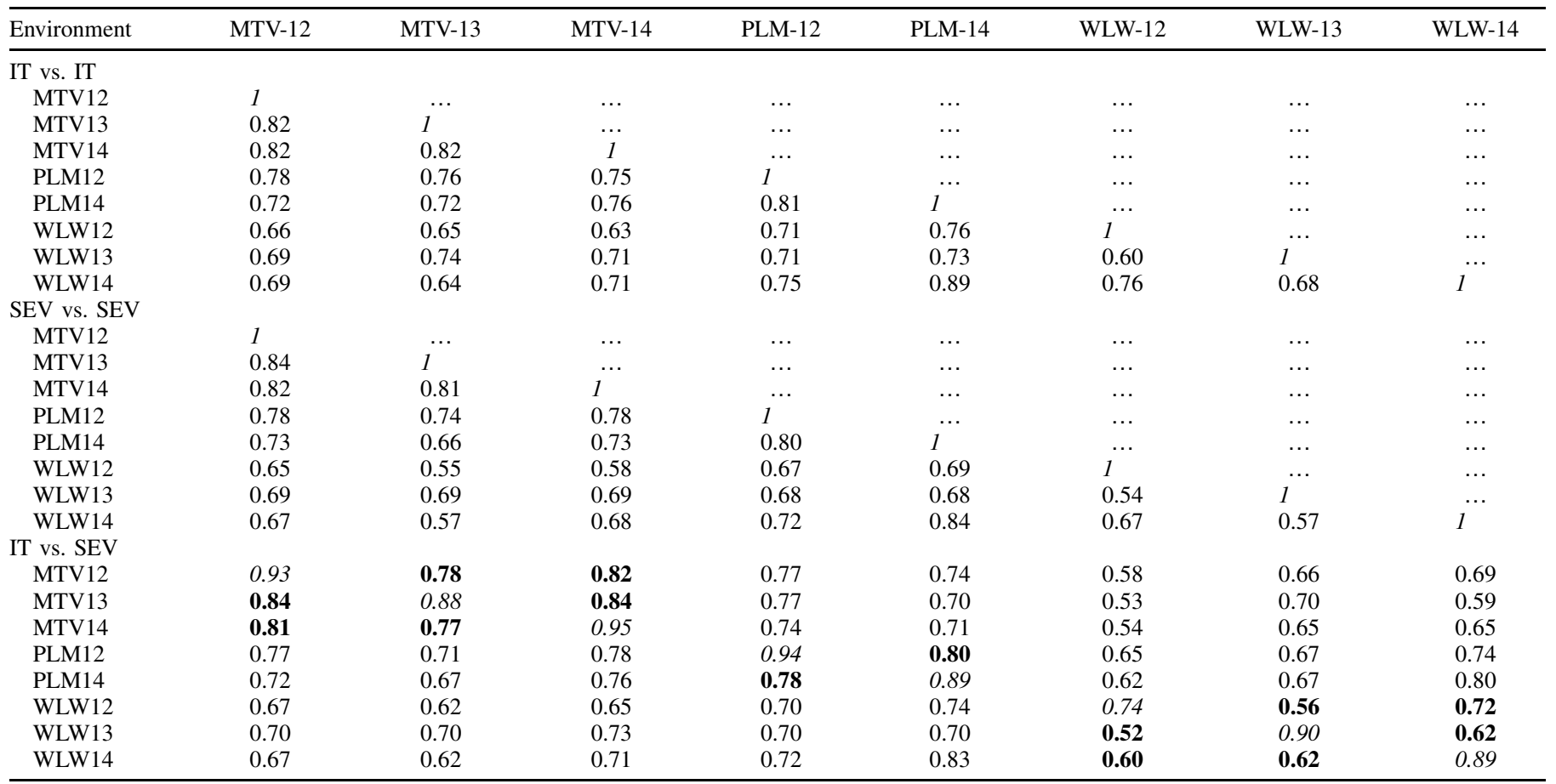

a Data in italics indicate comparisons between the same location and year and in bold indicate comparisons between different years in the same location. All correlation coefficients are highly significant $(P<0.0001)$. Locations: MTV $=$ Mount Vernon, WA; PLM $=$ Spillman Farm, Pullman, WA; and WLW $=$ Whitlow Farm, Pullman, WA; followed by year. 
locus was significantly associated with stripe rust resistance in both scores and BLUE values. The other 6B locus, QYr.tsw-6B.2, was tagged by SNP marker IWB74027 and was also associated with seedling resistance to PSTV-4. In the field, this locus was associated with IT at MTV-12, PLM-12, PLM-14, WLW-13, and WLW-14 and BLUE-IT and with SEV at PLM-12, PLM-14, WLW-14, and BLUESEV. The $R^{2}$ values of the two 6B loci ranged from 0.03 to 0.06 for IT and from 0.04 to 0.05 for SEV. A single locus on chromosome 6D, QYr.tsw-6D, was tagged by SNP marker IWB6427 and located at the $101.63 \mathrm{cM}$. For IT $\left(R^{2}=0.03\right)$, this locus was significant at MTV-12, MTV-13, PLM-12, and WLW-12 and BLUE-IT; and, for SEV $\left(R^{2}=\right.$ 0.04), at MTV-12, MTV-13, MTV-14, PLM-12, PLM-14, and WLW14 and BLUE-SEV. QYr.tsw-7A.2 was tagged by SNP marker IWB44026 located at $183.22 \mathrm{cM}$ on the long arm of chromosome 7A. This locus was associated with IT $\left(R^{2}=0.03\right)$ at MTV-12 and MTV-13 and SEV $\left(R^{2}=0.04\right)$ at MTV-12, MTV-13, MTV-14, WLW-12, and WLW-14 and BLUE-SEV. The favorable alleles for QYr.tsw-5D, QYr.tsw-6B.4, QYr.tsw-6D, and QYr.tsw-7A.2 were present at high frequencies in the panel and across the two subpopulations. Very few genotypes carry the favorable allele of QYr.tsw-6B.2.

Some genotypes carried the resistance allele for $\operatorname{Lr} 34 / \mathrm{Yr} 18$ but the KaspLr34/Yr18 marker was significantly associated with IT in one environment (MTV13) and SEV in four of the eight environments (MTV12, MTV13, MTV14, and PLM14). Yr15-linked SSR marker barc8 was associated with IT in six environments (MTV12, MTV13, MTV14, PLM12, WLW1,2 and WLW13) and SEV in seven environments (MTV12, MTV13, MTV14, PLM12, WLW12, WLW13, and WLW14), whereas the KaspSr28 was significantly associated with stripe rust IT and SEV in all environments (MTV12, MTV13, MTV14, PLM12, PLM14, WLW12, WLW13, and WLW14).
Effect of pyramiding QTL for adult plant resistance. The estimated number of favorable alleles in each specific genotype was classified based on marker haplotypes to assess the cumulative effect of favorable MTA on stripe rust response. The number of resistance-associated MTA present in a specific genotype ranged from 5 to 23. The variety Alikat had the fewest estimated favorable alleles while varieties UC1682 and Clear White 515 had the highest estimated number of resistance alleles. In general, accumulation of resistance-associated alleles was strongly correlated with actual stripe rust resistance response. Genotypes estimated to have $<10$ favorable alleles (average IT $=6.43$ and $\mathrm{SEV}=61.43$ ) were more susceptible than those estimated to carry $>20$ favorable alleles (IT $=$ 1.25 and SEV = 2.73). This direct relationship was further supported by the linear regression coefficients for IT $\left(R^{2}=-0.45, P<0.0001\right)$ and $\operatorname{SEV}\left(R^{2}=-5.22, P<0.0001\right)$ (Fig. 3). Furthermore, variation in the number of favorable alleles for these 25 loci explained 51 and $54 \%$ of variation in IT and SEV responses to stripe rust, respectively.

Distribution of favorable alleles for adult plant resistance across breeding programs. On average, genotypes from the University of Saskatchewan-Alberta and the University of IdahoAberdeen had the fewest $(n=8)$ estimated number of favorable alleles while genotypes from the University of California-Davis (UCD) carried the highest $(n=15)$ estimated number of favorable alleles (Supplementary Fig. S5). There was a strong correspondence between the average estimated number of favorable alleles in the breeding programs and the level of resistance to stripe rust (Supplementary Fig. S6), with UCD germplasm having the lowest IT and SEV across eight environments. Similarly, CIMMYT germplasm also had lower IT and SEV compared with other breeding programs with fewer estimated favorable alleles.

TABLE 3. Loci significantly associated with all-stage response to races of PSTV-4 and PSTv-14 in the 237 elite spring wheat genotypes

\begin{tabular}{|c|c|c|c|c|c|c|c|c|}
\hline \multirow[b]{2}{*}{$\mathrm{QTL}^{\mathrm{a}}$} & \multirow[b]{2}{*}{ Markerb } & \multirow[b]{2}{*}{ Chr. $^{\mathrm{c}}$} & \multirow[b]{2}{*}{$\mathrm{cM}^{\mathrm{d}}$} & \multirow[b]{2}{*}{ Allele } & \multirow[b]{2}{*}{$\mathrm{MAF}^{\mathrm{f}}$} & \multirow[b]{2}{*}{ Stat $^{\mathrm{g}}$} & \multicolumn{2}{|c|}{ Race } \\
\hline & & & & & & & PSTv-4 & PSTv-14 \\
\hline \multirow{2}{*}{ QYr.tsw-1A.1 } & IWB12795 & $1 \mathrm{~A}$ & 29.11 & $\underline{\mathrm{T}} / \mathrm{C}$ & 0.39 & $P$ & $6.24 \mathrm{E}-04$ & - \\
\hline & $\ldots$ & $\ldots$ & $\ldots$ & $\overline{-}$ & $\ldots$ & $R^{2}$ & 0.04 & - \\
\hline \multirow[t]{2}{*}{ QYr.tsw-1A.2 } & IWB20633 & $1 \mathrm{~A}$ & 48.95 & $\underline{\mathrm{T}} / \mathrm{C}$ & 0.32 & $P$ & $5.29 \mathrm{E}-04$ & - \\
\hline & $\ldots$ & $\ldots$ & $\ldots$ & $-\ldots$ & $\ldots$ & $R^{2}$ & 0.04 & - \\
\hline \multirow{2}{*}{ QYr.tsw-1A.3 } & IWB56353 & $1 \mathrm{~A}$ & 83.70 & $\mathrm{~A} / \underline{\mathrm{G}}$ & 0.42 & $P$ & 7.37E-04 & - \\
\hline & $\ldots$ & $\ldots$ & $\ldots$ & $\ldots$ & $\ldots$ & $R^{2}$ & 0.04 & - \\
\hline \multirow[t]{2}{*}{ QYr.tsw-1B.1 } & IWB34847 & $1 \mathrm{~B}$ & 31.09 & $\underline{\mathrm{A} / \mathrm{G}}$ & 0.48 & $P$ & - & $9.11 \mathrm{E}-04$ \\
\hline & $\ldots$ & $\ldots$ & $\ldots$ & $-\ldots$ & $\ldots$ & $R^{2}$ & - & 0.04 \\
\hline \multirow{2}{*}{$\cdots$} & $\operatorname{Yr} 15$ & $1 \mathrm{~B}$ & $\ldots$ & $\ldots$ & 0.07 & $P$ & $2.08 \mathrm{E}-05$ & $9.65 \mathrm{E}-05$ \\
\hline & & $\ldots$ & $\ldots$ & $\ldots$ & $\ldots$ & $R^{2}$ & 0.06 & 0.06 \\
\hline \multirow[t]{2}{*}{ QYr.tsw-1D } & IWB35520 & 1D & 89.58 & $\mathrm{~A} / \underline{\mathrm{C}}$ & 0.15 & $P$ & - & $2.24 \mathrm{E}-04$ \\
\hline & $\ldots$ & $\ldots$ & $\ldots$ & $\ldots$ & $\ldots$ & $R^{2}$ & - & 0.05 \\
\hline \multirow[t]{2}{*}{ QYr.tsw-2A.1 } & IWB1593.1 & $2 \mathrm{~A}$ & 123.65 & $\mathrm{~A} / \underline{\mathrm{G}}$ & 0.30 & $P$ & $8.93 \mathrm{E}-05$ & - \\
\hline & $\ldots$ & $\ldots$ & $\ldots$ & $\ldots$ & $\ldots$ & $R^{2}$ & 0.05 & - \\
\hline \multirow[t]{2}{*}{ QYr.tsw-2A.2 } & IWB10956 & $2 \mathrm{~A}$ & 139.85 & $\underline{\mathrm{A} / \mathrm{G}}$ & 0.18 & $P$ & - & 2.67E-04 \\
\hline & & $\ldots$ & $\ldots$ & $\ldots$ & $\ldots$ & $R^{2}$ & - & 0.05 \\
\hline \multirow[t]{2}{*}{$\cdots$} & $\operatorname{Sr} 28$ & $2 \mathrm{~B}$ & $\ldots$ & $\ldots$ & 0.05 & $P$ & - & $8.26 \mathrm{E}-04$ \\
\hline & & $\ldots$ & $\ldots$ & $\ldots$ & $\ldots$ & $R^{2}$ & - & 0.04 \\
\hline \multirow[t]{2}{*}{ QYr.tsw-2B.1 } & IWB32165 & $2 \mathrm{~B}$ & 109.24 & $\mathrm{~T} / \underline{\mathrm{C}}$ & 0.13 & $P$ & $6.56 \mathrm{E}-04$ & $2.88 \mathrm{E}-04$ \\
\hline & & $\ldots$ & $\ldots$ & $\ldots$ & $\ldots$ & $R^{2}$ & 0.04 & 0.05 \\
\hline \multirow[t]{2}{*}{ QYr.tsw-3A.1 } & IWB26833 & $3 \mathrm{~A}$ & 59.01 & $\underline{\mathrm{A} / \mathrm{G}}$ & 0.41 & $P$ & $6.00 \mathrm{E}-04$ & - \\
\hline & & $\ldots$ & $\ldots$ & $-\ldots$ & $\ldots$ & $R^{2}$ & 0.04 & - \\
\hline \multirow[t]{2}{*}{ QYr.tsw-5A } & IWA3530 & $5 \mathrm{~A}$ & 39.62 & $\mathrm{~A} / \underline{\mathrm{G}}$ & 0.32 & $P$ & $2.19 \mathrm{E}-04$ & - \\
\hline & $\ldots$ & $\ldots$ & $\ldots$ & $\ldots$ & $\ldots$ & $R^{2}$ & 0.05 & - \\
\hline \multirow[t]{2}{*}{ QYr.tsw-5B } & IWA7815 & $5 B$ & 71.64 & $\underline{\mathrm{A}} / \mathrm{G}$ & 0.33 & $P$ & - & $2.20 \mathrm{E}-11$ \\
\hline & & $\ldots$ & $\ldots$ & $\ldots$ & . & $R^{2}$ & - & 0.19 \\
\hline \multirow[t]{2}{*}{ QYr.tsw-6B.1 } & IWB25986 & $6 \mathrm{~B}$ & 57.07 & $\mathrm{~T} / \underline{\mathrm{C}}$ & 0.16 & $P$ & $3.42 \mathrm{E}-04$ & - \\
\hline & & $\cdots$ & $\ldots$ & $\ldots$ & 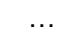 & $R^{2}$ & 0.04 & - \\
\hline \multirow[t]{2}{*}{ QYr.tsw-6B.2 } & IWB74027 & $6 \mathrm{~B}$ & 66.08 & $\underline{\mathrm{T}} / \mathrm{C}$ & 0.05 & $P$ & - & 4.92E-04 \\
\hline & $\cdots$ & $\ldots$ & $\ldots$ & $\ldots$ & $\ldots$ & $R^{2}$ & - & 0.05 \\
\hline
\end{tabular}

a Name of putative quantitative trait loci (QTL) identified by significant markers from marker-trait associations.

b Markers associated with all-stage resistance to PSTV-4 and PSTV-14.

c Chromosome location of markers according to Wang et al. (2014).

d Chromosome position of markers in centimorgans (cM) according to Wang et al. (2014).

e Alleles with underline signify resistant alleles.

f Minor allele frequency.

g $P=$ marker-wise $P$ value and $R^{2}=$ percentage of phenotypic variation explained by the marker. 


\section{DISCUSSION}

Phenotypic variation for stripe rust response among TCAP elite lines. In this study, we used a panel consisting of elite North American spring wheat genotypes from 10 public wheat-breeding institutions in Mexico, the United States, and Canada. The panel was mainly composed of well-adapted, advanced and elite breeding lines and cultivars; hence, there were minimal differences in major developmental traits such as plant height and heading date (J. Godoy, S. Gizaw, S. Chao, N. Blake, A. Carter, R. Cuthbert, J. Dubcovsky, P. Hucl, K. Kephart, C. Pozniak, V. Vara Prasad, M. Pumphrey, and L. Talbert, unpublished data) yet the panel displayed a wide spectrum of diversity for response to stripe rust in both seedling and adult-plant stages. This result makes the panel ideal to accurately evaluate reaction to stripe rust and identify MTA that are not confounded with developmental traits. The bimodal distribution of IT in seedling stage screening experiments indicated that the trait was controlled by only a few loci, further supporting reports of major genes influencing disease response when infections occur during this stage. The generally normal distribution and increase in resistance at adultplant stages across environments showed the presence of effective APR QTL in the panel. Analysis of variance also indicated the availability of significant variation among the genotypes in the panel. Significant correlations among traits showed the consistency of data generated across environments, which was also confirmed by the high heritability estimates, indicating a repeatable and reliable dataset for GWAS.
LD and population structure. In this panel, genome-wide LD decay was estimated at approximately $3 \mathrm{cM}$, which was consistent with that reported by Chao et al. (2010) on a larger population of elite spring wheat cultivars from the United States. Given the average genomewide marker coverage of $0.4 \mathrm{cM} / \mathrm{SNP}$ (J. Godoy, S. Gizaw, S. Chao, N. Blake, A. Carter, R. Cuthbert, J. Dubcovsky, P. Hucl, K. Kephart, C. Pozniak, V. Vara Prasad, M. Pumphrey, and L. Talbert, unpublished data), the number of markers ensured good coverage for GWAS. The significant effect of population structure on stripe rust response is likely due to stripe rust being more prevalent and damaging in wheat production areas in the western United States and Mexico.

Marker-trait associations for race-specific or seedling resistance. Marker-trait associations for race-specific or seedling resistance were detected in chromosomes $1 \mathrm{~A}, 1 \mathrm{~B}, 1 \mathrm{D}, 2 \mathrm{~A}, 2 \mathrm{~B}, 3 \mathrm{~A}$, $5 \mathrm{~A}, 5 \mathrm{~B}, 6 \mathrm{~B}$, and 7B (Table 3). The SNP markers associated with resistance at a seedling stage on $3 \mathrm{~A}($ QYr.tsw-3A.1) and 6B (QYr.tsw-6B.2) were also detected at the adult stage. In all, 10 of 13 MTA identified in this study were in close proximity to previously reported stripe rust resistance genes or QTL. Three MTA were potentially new loci associated with race-specific all-stage resistance.

Chromosome group 1. Due to their proximity, the three distinct chromosome 1A loci may be either identical or closely linked to three previously reported QTL identified through biparental QTL mapping. LD analysis showed further evidence that they were unlinked. The LD values $\left(R^{2}\right)$ for IWB12795 and IWB56353 were 0.09 and 0.00005 ,

TABLE 4. Loci significantly associated with infection type (IT) or severity (SEV) in at least four environments with marker-wise $P<0.01$ and false discovery rate $($ FDR $)<0.01$ in at least one environment ${ }^{\mathrm{a}}$

\begin{tabular}{|c|c|c|c|c|c|c|c|c|c|c|c|}
\hline Marker & IWB11136 & IWB24342 & IWB65219 & IWB34480 & IWB11792 & IWB36635 & IWA2821 & IWB45887 & IWB74027 & IWB6427 & IWB44026 \\
\hline $\begin{array}{l}\text { Putative QTL } \\
\text { name }\end{array}$ & $\begin{array}{c}\text { QYr.tsw- } \\
2 A .3\end{array}$ & $\begin{array}{c}\text { QYr.tsw- } \\
2 A .4\end{array}$ & $\begin{array}{c}\text { QYr.tsw- } \\
2 A .5\end{array}$ & $\begin{array}{c}\text { QYr.tsw- } \\
2 B .2\end{array}$ & $\begin{array}{c}\text { QYr.tsw- } \\
2 D\end{array}$ & $\begin{array}{c}\text { QYr.tsw- } \\
\quad 3 A .2\end{array}$ & $\begin{array}{l}\text { QYr.tsw- } \\
5 D\end{array}$ & $\begin{array}{c}\text { QYr.tsw- } \\
6 B .4\end{array}$ & $\begin{array}{c}\text { QYr.tsw- } \\
\quad 6 B .2\end{array}$ & $\begin{array}{c}\text { QYr.tsw- } \\
6 D\end{array}$ & $\begin{array}{l}\text { QYr.tsw- } \\
\quad 7 A .2\end{array}$ \\
\hline Chromosome & $2 \mathrm{AS}$ & 2AS & $2 \mathrm{AS}$ & 2BS & 2DS & 3AL & 5DL & 6BS & 6BS & 6DL & 7AL \\
\hline Position $^{b}$ & 9.41 & 25.97 & 48.44 & 19.95 & 6.75 & 123.01 & 190.80 & 57.07 & 66.08 & 101.63 & 183.22 \\
\hline $\begin{array}{l}\text { Alleles (R } \\
\text { underlined) }\end{array}$ & $\underline{\mathrm{T}} / \mathrm{G}$ & $\underline{\mathrm{T}} / \mathrm{C}$ & $\mathrm{A} / \underline{\mathrm{C}}$ & $\mathrm{A} / \underline{\mathrm{G}}$ & $\mathrm{T} / \underline{\mathrm{C}}$ & $\underline{\mathrm{T}} / \mathrm{C}$ & $\mathrm{T} / \underline{\mathrm{C}}$ & $\mathrm{A} / \underline{\mathrm{G}}$ & $\underline{\mathrm{T}} / \mathrm{C}$ & $\mathrm{T} / \underline{\mathrm{C}}$ & $\underline{\mathrm{T}} / \mathrm{C}$ \\
\hline \multicolumn{12}{|l|}{ GWAS for $\mathrm{IT}^{\mathrm{c}}$} \\
\hline MTV-12 & $* *$ & $* * *$ & $* *$ & $* *$ & $* *$ & $* *$ & ns & $* * *$ & $* *$ & $* *$ & $* *$ \\
\hline MTV-13 & $* *$ & $* *$ & $* *$ & $* *$ & $* *$ & $* *$ & $* *$ & $* *$ & ns & $* *$ & $* *$ \\
\hline MTV-14 & $* * *$ & $* * *$ & $* *$ & $* * *$ & $* * *$ & $* * *$ & $* *$ & ns & ns & ns & ns \\
\hline PLM-12 & $* * * *$ & $* * * *$ & $* * * *$ & $* * * *$ & $* * * *$ & $* * * *$ & ns & $* * * *$ & $* *$ & $* *$ & ns \\
\hline PLM-14 & $* * * *$ & $* * * *$ & $* * * *$ & $* * * *$ & $* * * *$ & $* * * *$ & ns & $* *$ & $* *$ & ns & ns \\
\hline WLW-12 & $* * * *$ & $* * * *$ & $* * * *$ & $* * * *$ & $* * * *$ & $* * * *$ & ns & $* * * *$ & ns & $* *$ & ns \\
\hline WLW-13 & $* *$ & $* *$ & $* *$ & $* *$ & $* *$ & $* *$ & $* *$ & $* * *$ & $* *$ & ns & ns \\
\hline WLW-14 & $* * * *$ & $* * * *$ & $* * * *$ & $* * * *$ & $* * * *$ & $* * * *$ & ns & $* *$ & $* *$ & ns & ns \\
\hline BLUE_All & $* * * *$ & $* * * *$ & $* * *$ & $* * * *$ & $* * * *$ & $* * * *$ & $* *$ & $* * *$ & $* *$ & $* *$ & ns \\
\hline \multicolumn{12}{|l|}{ GWAS for SEV } \\
\hline MTV-12 & $* * * *$ & $* * * *$ & $* * * *$ & $* * * *$ & $* * * *$ & $* * * *$ & ns & $* * * *$ & ns & $* *$ & $* * * *$ \\
\hline MTV-13 & $* * *$ & $* * *$ & $* *$ & $* *$ & $* *$ & $* *$ & $* * * *$ & $* *$ & ns & $* * *$ & $* *$ \\
\hline MTV-14 & $* * *$ & $* * *$ & $* *$ & $* *$ & $* *$ & $* *$ & $* *$ & $* *$ & ns & $* *$ & $* *$ \\
\hline PLM-12 & $* * * *$ & $* * * *$ & $* * * *$ & $* * * *$ & $* * * *$ & $* * * *$ & $* *$ & $* *$ & $* * * *$ & $* * * *$ & ns \\
\hline PLM-14 & $* * * *$ & $* * * *$ & $* * * *$ & $* * * *$ & $* * * *$ & $* * * *$ & $* *$ & $* * *$ & $* *$ & $* *$ & ns \\
\hline WLW-12 & $* * * *$ & $* * * *$ & $* * * *$ & $* * * *$ & $* * * *$ & $* * * *$ & ns & ns & $* *$ & ns & $* *$ \\
\hline WLW-13 & $* * *$ & $* *$ & $* *$ & $* *$ & $* *$ & $* *$ & $* *$ & $* * *$ & ns & ns & ns \\
\hline WLW-14 & $* * * *$ & $* * * *$ & $* * * *$ & $* * * *$ & $* * * *$ & $* * * *$ & ns & $* *$ & $* *$ & $* *$ & $* *$ \\
\hline BLUE_All & $* * * *$ & $* * * *$ & $* * * *$ & $* * * *$ & $* * * *$ & $* * * *$ & $* * *$ & $* * * *$ & $* *$ & $* *$ & $* *$ \\
\hline \multicolumn{12}{|l|}{ GWAS seedling } \\
\hline Significant races & None & None & None & None & None & None & None & None & PSTV-14 & None & None \\
\hline \multicolumn{12}{|l|}{ Variation $(\%)^{\mathrm{d}}$} \\
\hline $\operatorname{IT}\left(R^{2}\right)$ & 0.08 & 0.07 & 0.08 & 0.08 & 0.08 & 0.09 & 0.04 & 0.06 & 0.03 & 0.03 & 0.03 \\
\hline $\operatorname{SEV}\left(R^{2}\right)$ & 0.12 & 0.12 & 0.12 & 0.12 & 0.12 & 0.12 & 0.05 & 0.05 & 0.04 & 0.04 & 0.04 \\
\hline \multicolumn{12}{|l|}{$\begin{array}{l}\text { Frequency of } \mathrm{R} \\
\text { allele } \mathrm{e}^{\mathrm{e}}\end{array}$} \\
\hline Overall & 0.07 & 0.08 & 0.07 & 0.07 & 0.07 & 0.07 & 0.17 & 0.79 & 0.05 & 0.78 & 0.35 \\
\hline Q1 (127 lines) & 0.13 & 0.13 & 0.13 & 0.13 & 0.13 & 0.12 & 0.17 & 0.84 & 0.06 & 0.94 & 0.47 \\
\hline Q2 (110 lines) & 0.01 & 0.01 & 0.01 & 0.01 & 0.01 & 0.01 & 0.16 & 0.72 & 0.04 & 0.61 & 0.21 \\
\hline
\end{tabular}

a Single-nucleotide polymorphism (SNP) indexes from the Illumina iSelect 90K wheat SNP assay (Wang et al. 2014).

b Scaled position from hexaploid wheat consensus map (Wang et al. 2014).

${ }^{c}$ Genome-wide association analysis (GWAS). Locations: MTV = Mount Vernon, WA; PLM = Spillman Farm, Pullman, WA; and WLW = Whitlow Farm, Pullman, WA. BLUE_All $=$ best linear unbiased estimate for all environments. Significance, marker-wise: *, **, and *** indicate $P<0.05,0.01$, and 0.001 , respectively; ***** indicates FDR $<0.01$; ns $=$ not significant.

d Percentage of phenotypic variation explained by the significant SNP. The maximum $R^{2}$ values for IT and SEV of each significant SNP in one of the environments in which it tested significant were used in the table.

${ }^{\mathrm{e}} \mathrm{Q} 1$ and Q2 are subpopulations. 
respectively, while IWB20633 and IWB56353 had an $R^{2}=0.0005$. The locus QYr.tsw-1A.1, identified by IWB12795 on chromosome 1A (29.11 cM), was within the confidence interval of the QYrtam-1A from Avocet, as reported by Basnet et al. (2014). Based on the integrated map, flanking markers wPt-671596 and wPt-2150 of QYr.tam-1A were just 2.75 $\mathrm{cM}$ (distal) and $1.36 \mathrm{cM}$ (proximal) from IWB12795, respectively. The locus QYr.tsw-1A.2, tagged by IWB20633 (48.95 cM) at the distal end of chromosome 1A, also fell within the confidence interval of a minor QTL QYr.sgi-1A. 1 detected from a DH population between Kariega $\times$ Avocet (Prins et al. 2011). The IWB20633 marker was within the QTL region at approximately $6.7 \mathrm{cM}$ distal to wPT-3698, which is the proximal flanking marker for QYr.sgi-1A.1. Another locus, QYr.tsw-1A.3, tagged by IWB56353 $(83.70 \mathrm{cM})$, was detected near the centromere of chromosome 1A. Bariana et al. (2010) reported a QTL, QYr.sun-1A, in this region from a DH population of Kukri $\times$ Janz. The QTL region spans at least $83 \mathrm{cM}$ and the SNP marker IWB56353 associated with QYr.tsw-1A.3 was positioned inside this region.

The QYr.tsw-1B.1 locus was tagged by IWB34847 $(31.09 \mathrm{cM})$ at the proximal end of the short arm region of chromosome 1B. This region of chromosome 1B harbors many $\mathrm{Yr}$ genes (namely, $\mathrm{Yr} 9$, Yr10, YrAlp, Yr15, YrH52, Yr64, Yr65, and Yr24/Yr26) (Maccaferri et al. 2015). However, based on the alignment of $\mathrm{Yr} 15, \mathrm{YrH} 52$, and Yr64 from Cheng et al. (2014), IWB34847 was positioned 21.68 and $26.15 \mathrm{cM}$ distal to Xbarc119 and Xgwm413, respectively, which flanked both $\mathrm{Yr} 15$ and $\mathrm{YrH} 52$. $\mathrm{Yr} 64$ was positioned $3.5 \mathrm{cM}$ proximal to Xgwm413 (Chen et al. 2014). QYr.tsw-1B.1 appears closer to $Q Y$ r. cau-1BS from Chinese winter wheat AQ24788-53 (Quan et al. 2013) and YrAlp from the spring wheat Alpowa (Lin and Chen 2007). The cultivars Penawawa and Louise carried the resistance allele for $Q Y r$. tsw-1B.1. Penawawa (Potam 70/Fielder) shares its pedigree with Alpowa (Fielder/Potam 70//Walladay/3/Walladay/Potam 70) while Wawawai (ID0001901//Potam 70/Fielder/5/Tifton 3725/Walladay// Fielder/Potam 70/3/N70003151/ID00065/4/ID000651/Potam 70), one of the parents of Louise, also has a pedigree similar to Alpowa. A Yr15-linked SSR marker was also significantly associated with IT for both PSTv-4 and PSTv-14. Yr15 is an all-stage resistance gene identified from wild emmer wheat (Triticum dicoccoides) that confers resistance to all known North American races of stripe rust (Murphy et al. 2009; Wan and Chen 2014; Wan et al. 2016).

Chromosome group 2. The locus QYr.tsw-2A.1, tagged by IWB1593.1 $(123.65 \mathrm{cM})$ on chromosome $2 \mathrm{~A}$, was within the confidence interval of the Yr32 from Carstens V reported by Eriksen et al. (2004). The resistance-associated allele was detected in $30 \%$ of the genotypes in the present study.

The locus QYr.tsw-2B.1 was detected at the proximal region of chromosome 2BL and tagged by IWB32165 (109.24 cM). This locus was significantly associated with IT to both PSTV-4 and PSTV-14. The position of IWB32165 on the integrated map falls
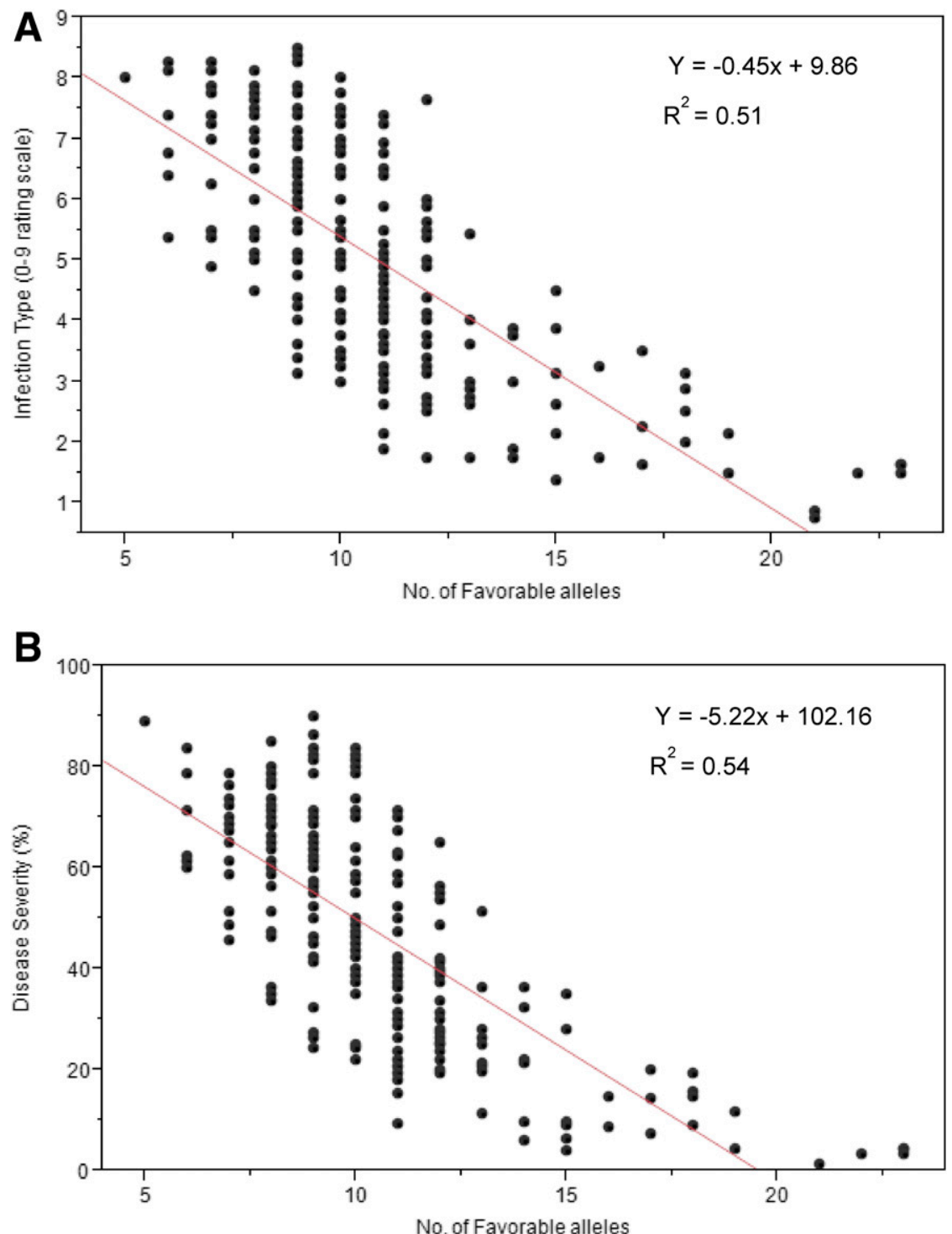

Fig. 3. Regression plots between the number of favorable alleles in each of the 237 genotypes and averaged A, infection type and $\mathbf{B}$, disease severity over eight different environments. Both regressions were highly significant $P<0.0001$. 
within the confidence interval of the QYrcaas-2BL QTL from German spring wheat Naxos (Ren et al. 2012) and QYraq.cau-2BL from Aquileja (Guo et al. 2008). The $\operatorname{Yr} 5$ gene is also located on chromosome 2BL. $\operatorname{Yr} 5$ is one of the major genes with rare virulence worldwide, except for $P$. striiformis f. sp. tritici races with virulence detected in Australia and India (Sharma-Poudyal et al. 2013; Zegeye et al. 2014). McGrann et al. (2014) reported a genetic map of $\operatorname{Yr} 5$ on the long arm of chromosome 2B with the marker wmc175 being $4.6 \mathrm{cM}$ proximal to the $\operatorname{Yr} 5$ gene. On the integrated map, IWB32165 was only $3.05 \mathrm{cM}$ proximal to wmc 175 . We ran kompetitive allele-specific polymerase chain reaction (KASP) markers flanking the $Y r 5$ gene and, based on the results, all lines that carried the resistance allele for $Y r 5$ also possessed the resistant allele for QYr.tsw-2B.1, but not vice versa. LD analysis $\left(R^{2}=0.03\right)$ between the KaspYr5 marker and IWB32165 showed that, despite their close proximity, they are not in strong LD.

Chromosome group 3. The locus QYr.tsw-3A.1, tagged by IWB26833 $(59.01 \mathrm{cM})$ at the distal end of chromosome $3 \mathrm{~A}$, was within the confidence interval of the QYrst.orr-3AL QTL from soft white winter wheat Stephens, as reported by Vazquez et al. (2012). Based on the integrated map, the closest locus wPt-1652 (36.89 cM) of QYr.tsw-3A.1 was $3.4 \mathrm{cM}$ from IWB26833 (40.29 cM). Stephens was widely grown and dominated the PNW acreage during the 1980s and 1990s. Among the cultivars from breeding programs in the states of Washington, Idaho, and California, $41 \%$ carried the resistanceassociated allele for QYr.tsw-3A.1.

Chromosome group 5. A locus on chromosome 5BL tagged by IWA7815, QYr.tsw-5B, was strongly associated $\left(R^{2}=0.19\right)$ with IT response to PSTv-14 at seedling stage. This locus may be related to the YrExp2 locus from spring wheat Express (Lin and Chen 2008). YrExp2 fell within the gwm604-to-gwm639 interval, and IWA7815 was positioned in the middle of this interval. Based on resistance gene analog polymorphism markers, Lin and Chen (2008) suggested that cultivars Expresso (developed from Express), Blanca Grande, and Buck Pronto may have YrExp2. Our panel also included Expresso and Blanca Grande and both carried the resistance allele for IWA7815 and were resistant to PSTv-14. Spring wheat varieties Louise, UI Cataldo, Lolo, Alturas, Otis, Jefferson, and Tara 2002 did not have the flanking markers for YrExp2 (Lin and Chen 2008). In our study, these cultivars carried the susceptible allele for IWA7815 and were susceptible to PSTv-14. However, YrExp2 is not effective against PSTv-14 but is effective against PSTv-4. SNP marker IWA7815 was also reported to be associated with all-stage resistance in a global winter wheat collection (Bulli et al. 2016).

Chromosome group 6. Two loci were identified in the short arm region of chromosome 6B: QYr.tsw-6B.1 (57.07 cM) and QYr.tsw$6 B .2(66.08 \mathrm{cM})$. Although only $9.01 \mathrm{cM}$ apart, LD analysis showed that the markers were not in $\mathrm{LD}$, with $R^{2}=0.005$. The first locus, QYr.tsw-6B.1, tagged by IWB25986 was within the confidence interval of QYr.sun-6B QTL (Bariana et al. 2010), QYr-6B (Suenaga et al. 2003), and $\operatorname{Yr} 36$ (Uauy et al. 2005). However, it was much closer to Yr36, an HTAP resistance gene from T. turgidum subsp. dicoccoides. Yr36 was reported to be completely linked to the barc101 SSR marker and IWB25986 was only $0.98 \mathrm{cM}$ proximal to barc101 based on the integrated map. This QTL region was also detected in the adult stage but the SNP associated with IT and SEV were different from that detected at seedling stage. The second locus, $Q Y$ r.tsw-6B.2, tagged by IWB74027 was within the confidence interval of QYr.caas-6BS (Lan et al. 2010). QYr.caas-6BS is within the interval barc136 to gwm361 and, based on the integrated map, IWB74027 also falls within this interval and $2.33 \mathrm{cM}$ from gwm361. Another QTL possibly at this locus is $Q Y r-6 B$, reported by William et al. (2006) from the CIMMYT cultivar Pavon 76. SSR marker gwm58, which was closest to the peak of $Q Y r-6 B$, was $2.24 \mathrm{cM}$ proximal to IWB74027.

Adult plant response in field screening nurseries. In total, 25 loci were significantly associated $(P<0.01$ in at least four environments) with IT and SEV but only 11 passed the FDR cutoff $<0.01$ in at least one environment and were considered highly significant. These 11 loci were identified in chromosomes 2A, 2B, 2D, 3A, 5D, 6B, 6D, and 7A (Table 4). Except for one locus on chromosome 6B, the remaining 10 loci were not detected in seedling GWAS analyses, indicating their possible role in APR. Yr15- and Sr28-linked markers were also associated with IT and SEV at adult-plant stages. The SSR marker barc8 linked to $\mathrm{Yr} 15$ was significant for both IT and SEV in all environments except PLM14, whereas KaspSr28 was significant in all environments for both disease scores. The significance of the locus tagged by KaspSr28 demonstrates a close association between $S r 28$ and a $Y r$ resistance gene or QTL such as $Y r 5$, Yr43, Yr44, Yr53, QYr.caas-2BL_Naxos, and QYraq.cau-2BL_ Aquileja (Guo et al. 2008; McGrann et al. 2014; Ren et al. 2012; Xu et al. 2013).

Chromosome group 2. A haplotype block of three loci on the short arm region of chromosome 2A, including QYr.tsw-2A.3 (9.41 cM)-QYr.tsw-2A.4 (25.97 cM)-QYr.tsw-2A.5 (48.44 cM), significantly associated with IT and SEV in all environments and BLUE values. Although the MTA were at least $16 \mathrm{cM}$ apart, LD analysis showed very high $R^{2}(0.94)$ among the three SNP markers. The genotypes in the panel carried either all three resistance-associated alleles or susceptible alleles at each locus. Only one genotype carried a different allelic combination. This chromosome region is known to carry the 2NS/AS translocation from $T$. ventricosum, a wild Triticeae species and the source of $\mathrm{Yr} 17 / \mathrm{Lr} 37 / \mathrm{Sr} 38$ resistance genes (Friebe et al. 1996). The 2NS/AS translocation, which is approximately 25 to $38 \mathrm{cM}$, was first introgressed into the winter bread wheat VPM1 and then deployed to different cultivars in California and Washington such as the winter wheat Madsen and Hyak and spring wheat Expresso. Of the 237 genotypes in our panel, only 17 carried this haplotype block, 16 of which came from breeding programs in the western United States and CIMMYT. Genetic analysis using the $\mathrm{Yrl7}$ (VENTRIUP-LN2) KASP marker confirmed the presence of the $\mathrm{Yr} 17$ resistance allele in all 16 genotypes. Although $\mathrm{Yr} 17$ is classified as an all-stage resistance gene, the QTL detected in this region were not associated with all-stage resistance in our greenhouse tests. A similar observation was reported by Naruoka et al. (2015) in a GWAS using an elite panel of winter wheat varieties from the PNW.

Aside from $\operatorname{Yr} 17$, other resistance loci reported on chromosome 2A include $Q Y$ r.tam-2AS from hard winter wheat TAM111, reported by Basnet et al. (2014); QYr.ucw-2A.2 identified by Maccaferri et al. (2015), using GWAS on a global spring wheat collection; and QYr.sun-2A (also designated as Yr56 in the wheat catalog) from durum wheat Wollaroi (Bansal et al. 2014). One of the flanking markers for QYr.tam-2AS, wPt-6431, is $4.16 \mathrm{cM}$ proximal to IWB11136 of QYr.tsw-2A.3. The SNP marker IWA422 for QYr.ucw-2A. 2 colocalized with IWB24342 for $Q Y$ r.tsw-2A.4 in the integrated map. The closest marker locus to QYr.sun-2A (wPt-4197) is $2.63 \mathrm{cM}$ distal to IWB24342 (QYr.tsw-2A.4).

QYr.tsw-2B.2, tagged by IWB34480 (34.74 cM) on the short arm of chromosome 2B, might be similar to QYrst.orr-2B.1 from soft white winter wheat Stephens, as reported by Vazquez et al. (2012). The DArT-associated marker wPt-5738 is $1.14 \mathrm{cM}$ distal from IWB34480. QTL QYr.inra-2BS from French winter wheat Renan was identified by Dedryver et al. (2009) and might also represent QYr.tsw-2B.1. The marker gwm210, positioned within the QTL region QYrinra- $2 B S$, is $0.36 \mathrm{cM}$ distal from IWB34480. The $2 \mathrm{~B}$ locus was effective against stripe rust in all environments and only a few genotypes, mostly from the western United States, carried the resistance allele.

The locus QYr.tsw-2D was detected on the short arm of chromosome 2D and tagged by IWB11792 $(14.2 \mathrm{cM})$. There are two reported QTL in this region: QYr.caas-2DS was detected from an Italian wheat Libellula (Lu et al. 2009) and Qyr.wpg-2D.1 was recently reported by Naruoka et al. (2015). Based on the integrated map, QYr.tsw-2D was much closer to the QYr.caas-2DS than Qyr.wpg-2D.1. SSR marker gwm261, one of the flanking markers for QYr.caas-2DS, is $1.2 \mathrm{cM}$ proximal to IWB11792 while IWA1939, the SNP associated 
with Qyrwpg-2D.1, is $11.9 \mathrm{cM}$ proximal to IWB11792. In total, 17 genotypes from Washington, California, CIMMYT, and Saskatchewan carried the resistance-associated allele for QYr.tsw-2D. QYr.tsw-2D was significantly associated with IT and SEV in all environments and may be a high priority for selection in breeding programs.

Chromosome group 6. Two loci associated with adult plant stripe rust responses were mapped in the short arm of chromosome 6B. LD analysis $\left(R^{2}=0.005\right)$ indicated that these two loci were not in LD. QYr.tsw-6B.4 was tagged by IWB45887 $(46.11 \mathrm{cM})$, and coincided with QYr.sun-6B from Australian wheat Janz (Bariana et al. 2010). One of the flanking markers for QYr.sun-6B, wPt-8183, was 12.15 cM proximal to IWB45887. However, QYr.tsw-6B.4 was aligned closest to the HTAP resistance gene Yr36 from T. turgidum subsp. dicoccoides (Uauy et al. 2005). IWB 45887 is also $0.98 \mathrm{cM}$ proximal to barc101, an SSR marker reported to be tightly linked to $\mathrm{Yr} 36$. This locus was also associated with race-specific seedling resistance to PSTV-4 but with a different SNP. The second locus, QYr.tsw-6B.2, tagged by IWB74027 (66.08 cM), is within the confidence interval of QYr.caas-6BS from Pingyuan 50 (Lan et al. 2010) and $Q Y r-6 B$ from Pavon 76 reported by William et al. (2006). One of the flanking markers closest to the QTL peak of QYr.caas-6BS, gwm361, is 2.33 cM proximal to IWB74027. The marker closest to the peak of $Q Y r$ $6 B$, gwm58, is $2.24 \mathrm{cM}$ proximal to IWB74027. QYr.tsw-6B.2 was also associated with all-stage resistance to PSTv-14.

One QTL was identified on the long arm of chromosome 6D. QYr.tsw-6D, tagged by IWB6427 (101.63 cM), was significant in fewer environments and $78 \%$ of the genotypes in the panel have the resistance-associated allele. QYr.tsw-6D is located near QYr.ucw-6D, identified by Maccaferri et al. (2015) from a global collection of spring wheat genotypes. The SNP IWA167 associated with QYr.ucw-6D is $4.22 \mathrm{cM}$ from IWB6427. In their article, $Q Y$ r.ucw- $6 D$ was present in the genotypes from the United States, indicating a possible connection with QYr.tsw-6D detected in the present study.

Newly documented resistance loci. Three SNP markers associated with loci on chromosomes 1D, 2A, and 5A are present in locations where all-stage resistance genes have not been reported previously. $Q Y$ r.tsw- $1 D$ on $1 \mathrm{D}$ was positioned close to the centromere at $89.58 \mathrm{cM}$. Four QTL have been previously reported on chromosome $1 \mathrm{D}$, and the most recent $(Q Y$ r.ucw-1D), reported by Maccaferri et al. (2015), was identified from a GWAS study using a global spring wheat collection. QYr.ucw- $1 D$ was closest to the centromere compared with the other QTL but was $41.3 \mathrm{cM}$ distal to $Q Y$ r.tsw-1D.

The QYrtsw-2A.2 (IWB10956) locus on the long arm of chromosome 2A falls between $\mathrm{Yr} 32$ and $\mathrm{Yrl}$ but outside their confidence intervals. The closest flanking marker to $\mathrm{Yrl}$, gwm311, was $29.64 \mathrm{cM}$ distal to IWB10956.

QYr.tsw-5A was located close to the tip of chromosome 5AS, and the closest published QTL (QYr.cau-5AS) was reported by Quan et al. (2013) from Chinese winter wheat AQ24788-53. However, QYr.tsw-5A was approximately $9 \mathrm{cM}$ from the peak (gwm705) of QYr.cau-5AS and was not within its confidence interval.

Three loci detected on chromosomes $3 \mathrm{~A}, 5 \mathrm{D}$, and $7 \mathrm{~A}$ are potentially newly documented APR loci because no other loci have been identified in close proximity based on the integrated map. The locus QYr.tsw-3A.2 was tagged by IWB36635 $(139.23 \mathrm{cM})$ on the long arm of chromosome 3A. QYr.cim-3A, identified by Rosewarne et al. (2012) from the cultivar Avocet, is $22.65 \mathrm{cM}$ from QYr.tsw-3A.2. The effect on $\mathrm{SEV}$ of $Q Y$ r.cim-3A was relatively small, ranging from 2.4 to $3.2 \%$, and effective in only half of the environments tested. QYr.tsw-3A.2 explained as much as 9 and $12 \%$ of the phenotypic variation for IT and SEV, respectively, and was effective in all environments. The resistance allele at QYr.tsw-3A.2 is present in 7\% of the genotypes in the panel, an indication of a potentially unique and underutilized source of resistance.

Chromosome 5D harbors only one reported stripe rust resistance gene, $\operatorname{Yr} 40$ (Kuraparthy et al. 2009). The $\mathrm{Yr} 40$ resistance gene is present in the T5DL.5DS-5MgS(0.95) wheat-alien translocation between common wheat and the wild relative Aegilops geniculata.
Although the translocation occurred on the short arm of $5 \mathrm{D}$, the locus $Q Y$ r.tsw-5D detected in this study was positioned at the long arm identified by IWA2821 $(166.86 \mathrm{cM})$ and was effective in $50 \%$ of the environments. No other $P$. striiformis $\mathrm{f}$. sp. tritici resistance loci have been reported on the long arm of chromosome 5D.

The locus QYr.tsw-7A.2, tagged by IWB44026 (183.22 cM) on the long arm of chromosome 7A, was positioned between Yrxyl (Zhou et al. 2011) and QYr.orr-7A (Vazquez et al. 2012). Considering the confidence intervals of the flanking markers and loci, QYr.tsw-7A is likely a new locus effective in adult plants. The DArT marker wPt-1023 associated with QYrorr-7A is $42.32 \mathrm{cM}$ distal to IWB44026, while the closest SSR marker barc49 to Yrxyl is $41 \mathrm{cM}$ from IWB44026.

Applications for breeding stripe rust-resistant cultivars. Genome-wide AM proved to be a powerful tool in detecting MTA for resistance to stripe rust at seedling and adult-plant stages. The alignment of some QTL with previous studies (i) served as a validation of the accuracy of our association analysis and (ii) offered an alternative marker system with improved mapping resolution compared with the large confidence intervals established in traditional biparental mapping efforts. The new QTL identified in this study are easily accessible, having come from well-adapted genotypes. Together, they can be stacked with other effective $Y r$ genes or QTL for a more durable resistance. We demonstrated the potential of pyramiding resistance-associated alleles to improve resistance to stripe rust. We also identified genotypes that carry more estimated resistance alleles that may be useful germplasm for breeding programs.

Compared with using a global or more diverse collection of genotypes, the use of an elite panel in this study provided us a limited array of alleles associated with stripe rust resistance. However, the panel gave a good picture of the current diversity of resistance genes in North American spring wheat. All highly significant loci for $P$. striiformis $\mathrm{f}$. sp. tritici response in adult plants identified in this study were carried mostly by cultivars from breeding programs in the western United States and CIMMYT. Consequently, cultivars that carry more resistance alleles also came from this region. The wheat-breeding community can use these available resources to enrich the current gene pool and further improve breeding efforts to combat stripe rust.

\section{ACKNOWLEDGMENTS}

This material is based upon work that is supported by the United States Department of Agriculture (USDA) National Institute of Food and Agriculture under awards 2017-67007-25939, 2016-68004-24770, and 2011-68002-30029. Lines for the AM panel were contributed by J. Chen (University of Idaho), K. Glover (South Dakota State University), J. Dubcovsky (UCD), M. Pumphrey (Washington State University), J. Anderson (University of Minnesota), P. Hucl (University of Saskatchewan), C. Pozniak (University of Saskatchewan), L. Talbert (Montana State University), D. Spaner (University of Alberta), G. Humphries (Agriculture and Agri-Food Canada), R. DePauw (Agriculture and Agri-Food Canada), R. Knox (Agriculture and Agri-Food Canada), and S. Fox (Agriculture and Agri-Food Canada). We thank our colleagues at the USDA Agricultural Research Service Small Grains Genotyping Laboratories and CIMMYT.

\section{LITERATURE CITED}

Bajgain, P., Rouse, M. N., Bulli, P., Bhavani, S., Gordon, T., Wanyera, R., Njau, P. N., Legesse, W., Anderson, J. A., and Pumphrey, M. O. 2015. Association mapping of North American spring wheat breeding germplasm reveals loci conferring resistance to Ug99 and other African stem rust races. BMC Plant Biol. 15:249.

Bansal, U. K., Kazi, A. G., Singh, B., Hare, R. A., and Bariana, H. S. 2014. Mapping of durable stripe rust resistance in a durum wheat cultivar Wollaroi. Mol. Breed. 33:51-59.

Bariana, H., Bansal, U., Schmidt, A., Lehmensiek, A., and Kaur, J. 2010. Molecular mapping of adult plant stripe rust resistance in wheat and identification of pyramided QTL genotypes. Euphytica 176:251-260.

Basnet, B. R., Singh, R. P., Ibrahim, A. M. H., Herrera-Foessel, S. A., HuertaEspino, J., Lan, C., and Rudd, J. C. 2014. Characterization of Yr54 and other genes associated with adult plant resistance to yellow rust and leaf rust in common wheat Quaiu 3. Mol. Breed. 33:385-399. 
Breseghello, F., and Sorrells, M. 2006. Association mapping of kernel size and milling quality in wheat (Triticum aestivum L.) cultivars. Genetics 172: 1165-1177.

Bulli, P., Zhang, J., Chao, S., Chen, X. M., and Pumphrey, M. 2016. Genetic architecture of resistance to stripe rust in a global winter wheat germplasm collection. G3: Genes Genomes Genet. 7:2237-2253.

Cavanagh, C. R., Chao, S., Wang, S., Huang, B. E., Stephen, S., Kiani, S., Forrest, K., Saintenac, C., Brown-Guedira, G. L., Akhunova, A., See, D., Bai, G., Pumphrey, M., Tomar, L., Wong, D., Kong, S., Reynolds, M., Lopez de Silva, M., Bockelman, H., Talbert, L., Anderson, J. A., Dreisigacker, S., Baenziger, S., Carter, A., Korzun, V., Morrell, P. L., Dubcobsky, J., Morrell, M. K., Sorrells, M. E., Hayden, M. J., and Akhunov, E. 2013. Genome-wide comparative diversity uncovers multiple targets of selection for improvement in hexaploid wheat landraces and cultivars. Proc. Natl. Acad. Sci. USA 110:8057-8062.

Chao, S., Dubcovsky, J., Dvorak, J., Luo, M. C., Baenziger, S., Matnyazov, R., Clark, D., Talbert, L., Anderson, J., Dreisigacker, S., Glover, K., Chen, J., Campbell, K., Bruckner, P., Rudd, J., Haley, S., Carver, B., Perry, S., Sorrells, M., and Akhunov, E. 2010. Population- and genome-specific patterns of linkage disequilibrium and SNP variation in spring and winter wheat (Triticum aestivum L.). BMC Genomics 11:727.

Chen, W. Q., Wellings, C., Chen, X. M., Kang, Z. S., and Liu, T. G. 2014. Wheat stripe (yellow) rust caused by Puccinia striiformis f. sp. tritici. Mol. Plant Pathol. 15:433-446.

Chen, X. M. 2005. Epidemiology and control of stripe rust (Puccinia striiformis f. sp. tritici) on wheat. Can. J. Plant Pathol. 27:314-337.

Chen, X. M. 2013. Review article: High-temperature adult-plant resistance, key for sustainable control of stripe rust. Am. J. Plant Sci. 4: Article ID 29148

Cheng, P., Xu, L. S., Wang, M. N., See, D. R., and Chen, X. M. 2014. Molecular mapping of genes Yr64 and Yr65 for stripe rust resistance in hexaploid derivatives of durum wheat accessions PI 331260 and PI 480016. Theor. Appl. Genet. 127:2267-2277.

Dedryver, F., Paillard, S., Mallard, S., Robert, O., Trottet, M., Nègre, S., Verplancke, G., and Jahier, J. 2009. Characterization of genetic components involved in durable resistance to stripe rust in the bread wheat 'Renan'. Phytopathology 99:968-973.

Earl, D. A., and vonHoldt, B. M. 2012. STRUCTURE HARVESTER: A website and program for visualizing STRUCTURE output and implementing the Evanno method. Conserv. Genet. Resour. 4:359-361.

Edae, E. A., Byrne, P. F., Haley, S. D., Lopes, M. S., and Reynolds, M. P. 2014. Genome-wide association mapping of yield and yield components of spring wheat under contrasting water regimes. Theor. Appl. Genet. 127:791-807.

Evanno, G., Regnaut, S., and Goudet, J. 2005. Detecting the number of clusters of individuals using the software STRUCTURE: A simulation study. Mol. Ecol. 14:2611-2620

Friebe, B., Jiang, J., Raupp, W., McIntosh, R., and Gill, B. 1996. Characterization of wheat-alien translocations conferring resistance to diseases and pests: Current status. Euphytica 91:59-87.

Guo, Q., Zhang, Z., Xu, T., Li, G., Feng, J., and Zhou, Y. 2008. Quantitative trait loci for high-temperature adult-plant and slow-rusting resistance to Puccinia striiformis f. sp. tritici in wheat cultivars. Phytopathology 98:803-809.

Jighly, A., Oyiga, B., Makdis, F., Nazari, K., Youssef, O., Tadesse, W., Abdalla, O., and Ogbonnaya, F. 2015. Genome-wide DArT and SNP scan for QTL associated with resistance to stripe rust (Puccinia striiformis $\mathrm{f}$. $\mathrm{sp}$. tritici) in elite ICARDA wheat (Triticum aestivum L.) germplasm. Theor. Appl. Genet. 128:1277-1295.

Kuraparthy, V., Sood, S., and Gill, B. 2009. Molecular genetic description of the cryptic wheat-Aegilops geniculata introgression carrying rust resistance genes $\mathrm{Lr} 57$ and $\mathrm{Yr} 40$ using wheat ESTs and synteny with rice. Genome 52: 1025-1036.

Lagudah, E. S., Krattinger, S. G., Herrera-Foessel, S., Singh, R. P., and HuertoEspino, J. 2009. Gene-specific markers for the wheat gene Lr34/Yr18/Pm38 which confers resistance to multiple fungal pathogens. Theor. Appl. Genet. 119:889-898.

Lan, C. X., Liang, S. S., Zhou, X. C., Zhou, G., Lu, Q. L., Xia, X. C., and He, Z. H. 2010. Identification of genomic regions controlling adult-plant stripe rust resistance in Chinese landrace Pingyuan 50 through bulked segregant analysis. Phytopathology 100:313-318.

Li, C., Chen, M., Chao, S., Yu, J., and Bai, G. 2013. Identification of novel gene, $H 34$, in wheat using recombinant inbred lines and single nucleotide polymorphism markers. Theor. Appl. Genet. 126:2065-2071.

Lin, F., and Chen, X. M. 2007. Genetics and molecular mapping of genes for race-specific all-stage resistance and non-race specific high-temperature adult-plant resistance to stripe rust in spring wheat cultivar Alpowa. Theor. Appl. Genet. 114:1277-1287.

Lin, F., and Chen, X. M. 2008. Molecular mapping of genes for race-specific overall resistance to stripe rust in wheat cultivars 'Express'. Theor. Appl. Genet. 116:797-806.
Line, R. F., and Chen, X. M. 1995. Successes in breeding for and managing durable resistance to wheat rusts. Plant Dis. 79:1254-1255.

Line, R. F., and Qayoum, A. 1992. Virulence, aggressiveness, evolution and distribution of races of Puccinia striiformis (the cause of stripe rust of wheat) in North America, 1968-1987. Tech. Bull. 1788. United State Department of Agriculture, Washington, DC.

Lipka, A. E., Tian, F., Wang, Q. S., Peiffer, J., Li, M., Bradbury, P. J., Gore, M. A., Bucker, E. S., and Zhang, Z. W. 2012. GAPIT: Genome association and prediction integrated tool. Bioinformatics 28:2397-2399.

Lu, Y. M., Lan, C. X., Linag, S. S., Zhou, X. C., Liu, D., Zhou, G., Lu, Q. L., Jing, J. X., Wang, M. N., Xia, X. C., and He, Z. H. 2009. QTL mapping for adult-plant resistance to stripe rust in Italian common wheat cultivars Libellula and Strampelli. Theor. Appl. Genet. 119:1349-1359.

Maccaferri, M., Zhang, J., Bulli, P., Abate, Z., Chao, S., Cantu, D., Bossolini, E., Chen, X. M., Pumphrey, M., and Dubcovsky, J. 2015. A genome-wide association study of resistance to stripe rust (Puccinia striiformis $\mathrm{f}$. sp. tritici) in a worldwide collection of hexaploid spring wheat collection (Triticum aestivum L.). G3: Genes Genomes Genet. 5:449-465.

Mago, R., Brown-Guedira, G., Dreisigacker, S., Breen, J., Jin, Y., Singh, R., Appels, R. S., Lagudah, E. S., Ellis, J., and Spielmeyer, W. 2011. An accurate DNA marker assay for stem rust resistance gene $\mathrm{Sr} 2$ in wheat. Theor. Appl. Genet. 122:735-744.

McGrann, G. R D., Smith, P. H., Burt, C., Mateos, G. R., Chama, T. N., MacCormack, R., Wessels, E., Agenbag, G., and Boyd, L. A. 2014. Genomic and genetic analysis of the wheat race-specific yellow rust resistance gene Yr5. J. Plant Sci. Mol. Breed. 3:2.

McIntosh, R., Dubcovsky, J., Rogers, J., Morris, C., Appels, R., and Xia, X. C. 2013. Catalogue of gene symbols for wheat. http://maswheat.ucdavis.edu/ CGSW/2013-2014_Supplement.pdf

McNeal, F., Konzak, C., Smith, E., Tate, W., and Russel, T. 1971. A uniform system for recording and processing cereal research data. USDA. Agricultural Research Service, Washington, DC.

Milus, E. A., and Line, R. F. 1986a. Number of genes controlling high-temperature, adult-plant resistance to stripe rust in wheat. Phytopathology 76:93-96.

Milus, E. A., and Line, R. F. 1986b. Gene action for inheritance of durable, high-temperature, adult-plant resistance to stripe rust in wheat. Phytopathology 76:435-441.

Murphy, L., Santra, D., Kidwell, K., Yan, G., Chen, X. M., and Campbell, K. 2009. Linkage maps of wheat stripe rust resistance genes and for use in marker-assisted selection. Crop Sci. 49:1786-1790.

Naruoka, Y., Ando, K., Bulli, P., Muleta, K. T., Rynearson, S., and Pumphrey, M. O. 2016. Identification and validation of SNP markers linked to the stripe rust resistance gene $\operatorname{Yr} 5$ in wheat. Crop Sci. 56:3055-3065.

Naruoka, Y., Garland-Campbell, K., and Carter, A. 2015. Genome-wide association mapping for stripe rust (Puccinia striiformis $\mathrm{f}$. sp. tritici) in US Pacific Northwest winter wheat (Triticum aestivum L.). Theor. Appl. Genet. 128:1083-1101.

Nordborg, M., and Weigel, D. 2008. Next-generation genetics in plants. Nature 456:720-723.

Prins, R., Pretorius, Z., Bender, C., and Lehmensiek, A. 2011. QTL mapping of stripe, leaf and stem rust resistance genes in a Kariega $\times$ Avocet $\mathrm{S}$ doubled haploid wheat population. Mol. Breed. 27:259-270.

Pritchard, J. K., Stephens, M., and Donnelly, P. 2000. Inference of population structure using multilocus genotype data. Genetics 155:945-959.

Qayoum, A., and Line, R. 1985. High-temperature, adult-plant resistance to stripe rust of wheat. Phytopathology 75:1121-1125.

Quan, W., Hou, G., Chen, J., Du, Z., and Lin, F. 2013. Mapping of QTL lengthening the latent period of Puccinia striiformis in winter wheat at the tillering growth stage. Eur. J. Plant Pathol. 136:715-727.

Ren, Y., Li, Z., He, Z., Wu, L., and Bai, B. 2012. QTL mapping of adult-plant resistances to stripe rust and leaf rust in Chinese wheat cultivar Bainong 64. Theor. Appl. Genet. 125:1253-1262.

Rosewarne, G., Herrera-Foessel, S., Singh, R., Huerta-Espino, J., Lan, C., and He, Z. 2013. Quantitative trait loci of stripe rust resistance in wheat. Theor. Appl. Genet. 126:2427-2449.

Rosewarne, G. M., Singh, R. P., Huerta-Espino, J., Herrera-Foessel, S. A., Forrest, K. L., Hayden, M. J., and Rebetzke, G. J. 2012. Analysis of leaf and stripe rust severities reveals pathotype changes and multiple minor QTLs associated with resistance in an Avocet $\times$ Pastor wheat population. Theor. Appl. Genet. 124:1283-1294.

Saitenac, C., Jiang, D., Wang, S., and Akhunov, E. 2013. Sequence-based mapping of the polyploid wheat genome. G3: Genes Genomes Genet. 3: 1105-1114.

SAS Institute Inc. 2007. JMP Genomics User Guide. SAS Press, Cary, NC.

Sharma-Poudyal, D., Chen, X. M., and Rupp, R. 2014. Potential oversummering and overwintering regions for the wheat stripe rust pathogen in the contiguous United States. Int. J. Biometeorol. 58:987-997.

Sharma-Poudyal, D., Chen, X. M., Wan, A. M., Zhan, G. M., Kang, Z. S., Cao, S. Q., Jin, S. L., Morgounov, A., Akin, B., Mert, Z., Shah, S. J. A., Bux, H., 
Ashraf, M., Sharma, R. C., Madariaga, R., Puri, K. D., Wellings, C., Xi, K. Q., Wanyera, R., Manninger, K., Ganzález, M. I., Koyda, M., Sanin, S., and Patzek, L. J. 2013. Virulence characterization of international collections of the wheat stripe rust pathogen, Puccinia striiformis f. sp. tritici. Plant Dis. 97:379-386.

Singh, D., Park, R., McIntosh, R., and Bariana, H. 2008. Characterization of stem rust and stripe rust seedling resistance genes in selected wheat cultivars from the United Kingdom. J. Plant Pathol. 90:553-562.

Somers, D., Isaac, P., and Edwards, K. 2004. A high-density microsatellite consensus map for bread wheat (Triticum aestivum L.). Theor. Appl. Genet. 109:1105-1114.

Song, Q., Shi, J., Singh, S., Fickus, E., Costa, J., Lewis, J., Gill, B., Ward, R., and Cregan, P. 2005. Development and mapping of microsatellite (SSR) markers in wheat. Theor. Appl. Genet. 110:550-560.

Suenaga, K., Singh, R., Huerta-Espino, J., and William, H. 2003. Microsatellite markers for genes $\operatorname{Lr} 34 / \mathrm{Yr} 18$ and other quantitative trait loci for leaf rust and stripe rust resistance in bread wheat. Phytopathology 93: 881-890.

Sukumaran, S., Dreisigacker, S., Lopes, M., Chavez, P., and Reynolds, M. P. 2015. Genome-wide association study for grain yield and related traits in an elite spring wheat population grown in temperate irrigated environments. Theor. Appl. Genet. 128:353-363.

Tabangin, M. E., Woo, J. G., and Martin, L. J. 2009. The effect of minor allele frequency on the likelihood of obtaining false positives. BMC Proc. 3:S41.

Uauy, C., Brevis, J., Chen, X., Khan, I., Jackson, L., Chicaiza, O., Distelfeld, A., Fahima, T., and Dubcovsky, J. 2005. High-temperature adult-plant (HTAP) stripe rust resistance gene Yr36 from Triticum turgidum ssp. dicoccoides is closely linked to the grain protein content locus Gpc-B1. Theor. Appl. Genet. 112:97-105.

Varella, A. C., Weaver, D. K., Sherman, J. D., Blake, N. K., Heo, H. Y., Kalous, J. R., Chao, S., Hofland, M. L., Martin, J. M., Kephart, K. D., and Talbert, L. E. 2015. Association analysis of stem solidness and wheat stem sawfly resistance in a panel of North American spring wheat germplasm. Crop Sci. 55:2046-2055.

Vazquez, M. D., Peterson, C. J., Riera-Lizarazu, O., Chen, X., Heesacker, A., Ammar, K., Crossa, J., and Mundt, C. C. 2012. Genetic analysis of adult plant, quantitative resistance to stripe rust in wheat cultivar "Stephens" in multi-environment trials. Theor. Appl. Genet. 124:1-11.

Wan, A. M., and Chen, X. M. 2014. Virulence characterization of Puccinia striiformis f. sp. tritici using a new set of $\mathrm{Yr}$ single-gene line differentials in the United States in 2010. Plant Dis. 98:1534-1542.
Wan, A. M., Chen, X. M., and Yuen, J. 2016. Races of Puccinia striiformis f. sp. tritici in the United States in 2011 and 2012 and comparison with races in 2010. Plant Dis. 100:966-975.

Wang, S., Wong, D., Forrest, K., Allen, A., Chao, S., Huang, B., Maccaferri, M., Salvi, S., Milner, S., Cattivelli, L., Mastrangelo, A., Whan, A., Stephen, S., Barker, G., Wieseke, R., Plieske, J., International Wheat Genome Sequencing Consortium, Lillemo, M., Mather, D., Appels, R., Dolferus, R., Brown-Guedira, G., Korol, A., Akhunova, A., Feuillet, C., Salse, J., Morgante, M., Pozniak, C., Luo, M., Dvorak, J., Morell, M., Dubcovsky, J., Ganal, M., Tuberosa, R., Lawley, C., Mikoulitch, I., Cavanagh, C., Edwards, K., Hayden, M., and Akhunov, E. 2014. Characterization of polyploid wheat genomic diversity using a high-density 90000 single nucleotide polymorphism array. Plant Biotechnol. J. 12:787-796.

Weir, B. 1996. Genetic Data Analysis II: Methods for Discrete Population Genetic Data. Sinauer Associates, Sunderland, MA.

William, H. M., Singh, R. P., Huerta-Espino, J., Suenaga, G., and Palacios, K. 2006. Characterization of genetic loci conferring adult plant resistance to leaf rust and stripe rust in spring wheat. Genome 49:977-990.

Xia, C. J., Wan, A. M., Wang, M. N., Jiwan, D. A., See, D. R., and Chen, X. M. 2016. Secreted protein gene derived-single nucleotide polymorphisms (SPSNPs) reveal population diversity and differentiation of Puccinia striiformis f. sp. tritici in the United States. Fungal Biol. 120:729-744.

Xiang, C., Feng, J. Y., Wang, M. N., Chen, X. M., See, D. R., Wan, A. M., and Wang, T. 2016. Molecular mapping of stripe rust resistance gene $Y r 76$ in winter club wheat cultivar Tyee. Phytopathology 106:1186-1193.

Xu, L. S., Wang, M. N., Cheng, P., Kang, Z. S., Hulbert, S. H., and Chen, X. M. 2013. Molecular mapping of Yr53, a new gene for stripe rust resistance in durum wheat accession PI 480148 and its transfer to common wheat. Theor. Appl. Genet. 126:523-533.

Yu, J., Pressoir, G., Briggs, W. H., Bi, I. V., Yamasaki, M., Doebley, J., and McMullen, M. D. 2006. A unified mixed-model method for association mapping that accounts for multiple levels of relatedness. Nat. Genet. 38:203-208.

Zegeye, H., Rasheed, A., Makdis, F., Badebo, A., and Ogbonnaya, F. 2014. Genome-wide association mapping for seedling and adult plant resistance to stripe rust in synthetic hexaploid wheat. PLoS One 9:e105593.

Zhou, X. L., Wang, W. L., Wang, L. L., Hou, D. Y., Jing, J. X., Wang, Y., Xu, Z. Q., Yao, Q., Yin, J. L., and Ma, D. F. 2011. Genetics and molecular mapping of genes for high-temperature resistance to stripe rust in wheat cultivar Xiaoyan 54. Theor. Appl. Genet. 123:431-438.

Zhu, C., Gore, M., Buckler, E. S., and Yu, J. 2008. Status and prospects of association mapping in plants. Plant Genome 1:5-20. 